\title{
FLAG: Fuzzy Logic Augmented Game Theoretic Hybrid Hierarchical Clustering Algorithm for Wireless Sensor Networks
}

Chandra Naik ( $\sim$ chandra.nitk2017@gmail.com )

National Institute of Technology Karnataka https://orcid.org/0000-0002-4760-3402

Pushparaj Shetty D

National Institute of Technology Karnataka

\section{Research Article}

Keywords: Wireless sensor networks, Game theory, Clustering, Fuzzy logic, Artificial intelligence, Optimization, Hybrid model

Posted Date: April 20th, 2021

DOl: https://doi.org/10.21203/rs.3.rs-323946/v1

License: (c) (i) This work is licensed under a Creative Commons Attribution 4.0 International License.

Read Full License

Version of Record: A version of this preprint was published at Telecommunication Systems on January 25th, 2022. See the published version at https://doi.org/10.1007/s11235-022-00878-2. 


\title{
FLAG: Fuzzy Logic Augmented Game Theoretic Hybrid Hierarchical Clustering Algorithm for Wireless Sensor Networks
}

\author{
Chandra Naik - Pushparaj Shetty D
}

Received: date / Accepted: date

\begin{abstract}
Stability of the Wireless Sensor Network(WSN) is the most critical factor in realtime and data-sensitive applications like military and surveillance systems. Many energy optimization techniques and algorithms have been proposed to extend the stability of a wireless sensor network. Clustering is a well regarded method in the research communities among them. Hence, this paper presents hybrid hierarchical artificial intelligence based clustering techniques, named FLAG and I-FLAG. The first phase of these algorithms use gametheoretic technique to elect suitable cluster heads $(\mathrm{CHs})$ and later phase of the algorithms use fuzzy inference system to select appropriate super cluster heads( $\mathrm{SCHs})$ among CHs. The IFLAG is an improved version of FLAG where additional parameters like energy and distance are considered to elect CHs. Simulations are performed to check superiority of the proposed
\end{abstract}

\footnotetext{
Chandra Naik

Department of Mathematical and Computational Sciences, National Institute of Technology Karnataka, Surathkal,India

E-mail: chandra.nitk2017@gmail.com

Pushparaj Shetty D

Department of Mathematical and Computational Sciences, National Institute of Technology

Karnataka, Surathkal, India

E-mail: prajshetty@nitk.edu.in
}

algorithms over the existing protocols like LEACH, CHEF, and CROSS. Simulation results show that the average stability period of WSN is better in FLAG and I-FLAG compared to other protocols, and so is the throughput of WSN during the stability period.

Keywords Wireless sensor networks · Game theory · Clustering · Fuzzy logic · Artificial intelligence · Optimization · Hybrid model

\section{Introduction}

Recent advancement in microelectromechanical systems(MEMS) and wireless technology have enabled production of low power, inexpensive, and mutifunctional tiny devices with inbuilt communication, computing, and sensing capabilities known as sensor nodes. These sensor nodes forms a collaborative settings called a Wireless Sensor Network(WSN) which collects useful information from a field of interest [2]. Wireless sensor networks gained tremendous interest in a wide variety of applications like disaster management, home automation, traffic control, environment monitoring, agriculture, health care, and military target tracking. Generally, sensors are equipped with irreplaceable, limited capacity batteries, and deployed in a hostile or harsh environment to col- 
lect required information. Major part of these sensor nodes energy drained in data transmission and receipt. Thus, an energy management of sensors plays an important role in prolong lifetime of the WSNs. There are many methods proposed to achieve enery efficiency of the WSNs. Among them, one of the most influential technique is clustering, that divides the entire network into clusters. Each cluster has a cluster head $(\mathrm{CH})$ and many cluster members. The cluster head is responsible for collecting data from its members, fuse it, and forward to the base station. Many state-of-the-art algorithms are proposed for clustering in WSNs.

Game theory is a branch of mathematics and heavily used to analyze the problem of economics, social science, and computer science. In recent past, It is employed to solve clustering, routing, resource allocation, and coverage optimization problems in WSN [32]. The first game theoretic algorithm proposed for clustering in WSN is called CROSS(Clustered Routing for Selfish Sensors). Here, each sensor node act as a player and each node plays clustering game with other players within the network to decide whether to be $\mathrm{CH}$ or not. Later, the authors in [31] proposed localized algorithm by considering clustering game within close neighbors.

In the proposed work, hybrid game theory and fuzzy inference-based algorithms are proposed to improve the stability period of the network, named FLAG and I-FLAG. The FLAG protocol is a combination of CROSS and fuzzy inference system, where as I-FLAG is a hybrid of fuzzy inference system and improved version of CROSS protocol which considers energy of a sensor node and its distance to the BS as additional parameters.

The contributions of the paper are listed below:

- An improved version of CROSS game theoretic protocol for CHs selection.

- A fuzzy logic system is proposed to elect appropriate SCHs from CHs in FLAG and I-FLAG.
- Residual energy, centrality, and distance to base station are considered as member functions to compute SCH election probability.

- The proposed algorithms are compared with other clustering techniques like LEACH, CHEF, CROSS, and an improved CROSS.

The remaining parts of the paper is organized as follows. Section 2 discusses related work on clustering using state-of-the-art techniques. The network and radio model are briefed in Section 3. The detailed discussion of the proposed model is presented in Section 4. Results and discussion are summarized in Section 5 . Finally, Section 6 concludes the paper.

\section{Related Work}

Most famous clustering algorithm LEACH [6] ensures that every node plays the role of cluster head within a set time interval. Since all nodes turns to be $\mathrm{CHs}$ in rotation basis and therefore energy consumption is evenly distributed among nodes. However, random rotation leads to uneven distribution of the $\mathrm{CHs}$, and this leads to faster energy drain in certain nodes. As a improvement, LEACH-C (LEACH centralized) [5] algorithm is proposed. Here, each sensor node sends its location and energy information to the base station. Using Simulated Annealing algorithm and global information of nodes, BS selects optimal CHs. Since the algorithm expects each node to send information to the BS in every round, it causes lot of overhead and not suitable for larger networks. The Authors in [33] proposed HEED algorithm, which selects $\mathrm{CHs}$ periodically using residual energy and degree of nodes. The increase in network scalability and lifetime by supporting scalable data aggregation is observed in HEED compared to LEACH. However, it creates an isolated node in the network. A distributed energy efficient clustering(DEEC) algorithm is proposed in [23]. In this work, election of $\mathrm{CHs}$ based on ratio between residual energy of sensor node and average energy of the network. A stable election protocol(SEP) for heteroge- 
Table 1: Comparison between various methods

\begin{tabular}{lllll}
\hline $\begin{array}{l}\text { Protocol } \\
\text { Name }\end{array}$ & $\begin{array}{l}\text { Sensors } \\
\text { Heterogeneity }\end{array}$ & $\begin{array}{l}\text { Hierarchy or } \\
\text { Levels }\end{array}$ & $\begin{array}{l}\text { CH or SCH } \\
\text { Election method }\end{array}$ & $\begin{array}{l}\text { Goal (Extend } \\
\text { lifetime or stability ) }\end{array}$ \\
\hline LEACH [6] & No & 1-level & Random & lifetime \\
\hline LEACH-C [5] & No & 1-level & Random & Lifetime \\
\hline HEED [33] & No & 1-level & Random & Lifetime \\
\hline SEP [26] & Yes & 1-level & Random & Stability \\
\hline DEEC [23] & Yes & 1-level & Random & Lifetime \\
\hline CHEF [8] & No & 1-level & Fuzzy logic & Lifetime \\
\hline DE based [10] & No & 1-level & Heuristic & Lifetime \\
\hline GA based [7] & No & 1-level & Heuristic & Lifetime \\
\hline DUCF [3] & No & 1-level & Fuzzy logic & Lifetime \\
\hline FUCA [1] & No & 1-level & Fuzzy logic & Lifetime \\
\hline CROSS [9] & No & 1-level & Game theory & Lifetime \\
\hline LGCA [31] & No & 1-level & Game theory & Lifetime \\
\hline HGTD [32] & No & 1-level & Game theory & Lifetime \\
\hline FLAG & No & 2-level & Game theory and Fuzzy Logic & Stability \\
\hline
\end{tabular}

neous wireless sensor networks is proposed in [26]. This algorithm increases stability of the network and ensures reliability of the sensor networks for a longer time. Advancement in Artificial Intelligence(AI) has brought a different approach towards getting the solution to science and engineering problems. These include meta-heuristic algorithms, Fuzzy logic based algorithms, and game theory based algorithms.

Few of the meta-heuristic algorithms for clustering are described in $[7,10,11,19]$. The genetic algorithm based clustering algorithm is proposed in [7]. In [10], the authors used differential evolution algorithm for clustering. The authors in [11] proposed Biogeography-based optimization algorithm for clustering. This technique uses residual energy and distance to the base station for fitness function design. In [19], the authors adopted Biogeography-based optimization for clustering. This algorithm elects appropriate $\mathrm{CH}$ using residual energy, distance to base station, and distance to its member nodes. The authors in [4] proposed a hybrid meta-heuristic model for selecting load balanced cluster heads. In this work best features of Dif- ferential evolution and Artificial bee colony algorithms are exploited to get appropriate CHs. Further, Artificial bee algorithm is adopted for the dynamic re-localization of the mobile sink.

Many work in the literature adopts fuzzy logic technique for clustering. The authors in [8] proposed a fuzzy logic based CHEF algorithm. The algorithm considers remaining energy and local distance to select CHs. The sum of the distance from all the neighbor nodes within the radius $Q$ is referred as local distance in the algorithm. The authors in [18] proposed an unequal clustering approach using Fuzzy logic. Here, local density and distance to the base station are used as input to the fuzzy system to select competitive radius for cluster size. In DUCF [3] distance to base station, remaining energy, and node degree are used as input to the fuzzy inference engine in clustering process. In [14], the authors proposed a fuzzy logic based distributed clustering algorithm. This algorithm is designed by considering energy efficiency and coverage requirement of the network. It handles the hot spot problem by forming unequal sized clusters such that more $\mathrm{CH}$ are available near to 
the BS. The authors in [1] proposed a fuzzybased unequal clustering algorithm. The algorithm uses residual energy, distance to BS, and density as fuzzy input variables; Competition radius and rank as fuzzy output variables. The work in [28], uses fuzzy logic for clustering by considering residual energy in $\mathrm{CH}$, distance between $\mathrm{CH}$ and $\mathrm{BS}$, and degree of $\mathrm{CH}$. The authors in [20] adopted Fuzzy C-means algorithm for clustering in WSN. The authors in [13] adopted fuzzy logic to compute competition radius which decides the size of cluster for an elected $\mathrm{CH}$. The work considers distance to BS, residual energy, and node degree as fuzzy input variables to decide competition radius. The authors in [25], proposed a bacterial foraging optimization (BFO)-fuzzy method for multipath routing. The work in [21] adopted fuzzy logic system for unequal multihop clustering to extend lifetime of the network. The work in [15], uses fuzzy approach for unequal clustering. The authors consider energy level, distance to BS, neighbor density, and neighbor cost to decide cluster head and cluster radius. In this work, the authors used first two parameters for cluster head election and cluster radius computation, but last two parameters for cluster radius determination. In [16], the authors used a hybrid of flower pollination algorithm and fuzzy logic for clustering. Each node residual energy, node centrality, and distance to BS are used as input parameters to the fuzzy logic system for computing fitness of each node to elect appropriate $\mathrm{CH}$. The authors in [24] adopted fuzzy based clustering technique for effective load balancing and three tier multi-hop routing technique. In [17], the authors proposed a fuzzy logic integrated hierarchical LEACH algorithm. The algorithm consider mobility of sink, residual energy and centrality of $\mathrm{CH}$ to elect appropriate super cluster heads(SCHs), which is responsible for collecting data from $\mathrm{CH}$ and forwarding to the BS. In work [29], named FuzzyLEACH, the authors considered residual energy and position of sensor node as fuzzy descriptors to elect appropriate CH. In [30], the authors im- proved the work of [17] by proposing optimal LEACH clustering algorithm for electing right $\mathrm{CH}$, and used the energy average of $\mathrm{CHs}$ as a additional fuzzy input member function to elect appropriate $\mathrm{SCH}$.

Various game theoretic based clustering algorithms are proposed for WSNs. In [9], a first game theoretic algorithm is developed, followed by a work in [31], a localized game theoretical clustering algorithm (LGCA). An improved version of LGCA is proposed in [12] by adding energy component to select appropriate $\mathrm{CHs}$. The authors in [32], proposed a Hybrid, Game Theory based and Distributed clustering (HGTD). In this work, residual energy and the number of neighboring potential $\mathrm{CHs}$ are considered to elect final CHs. The authors in [22], proposed a coalition game theory based hierarchical routing protocol to ensure uniform energy consumption across the network. The authors in [27], proposed an energy-efficient clustering in mobile wireless sensor network using game theory. Here, a heterogeneous clustering game model is developed, in which predicted remaining energy, distance to base station, distance between nodes, and mobility speed of nodes are considered to compute probability inorder to elect right $\mathrm{CHs}$. Comparisons between few selected protocols and their goals are summarized in Table 1 .

\section{Network Model and Radio Model}

\subsection{Network Model}

The proposed network model consist of homogeneous nodes having equal energy, same sensing, and communicating capabilities as shown in Fig. 2. Initially, CHs are selected using game theoretic algorithm [9]. Secondly, SCHs are selected among CHs using fuzzy logic. The working procedure for proposed model is similar to the work in $[17,30]$. 


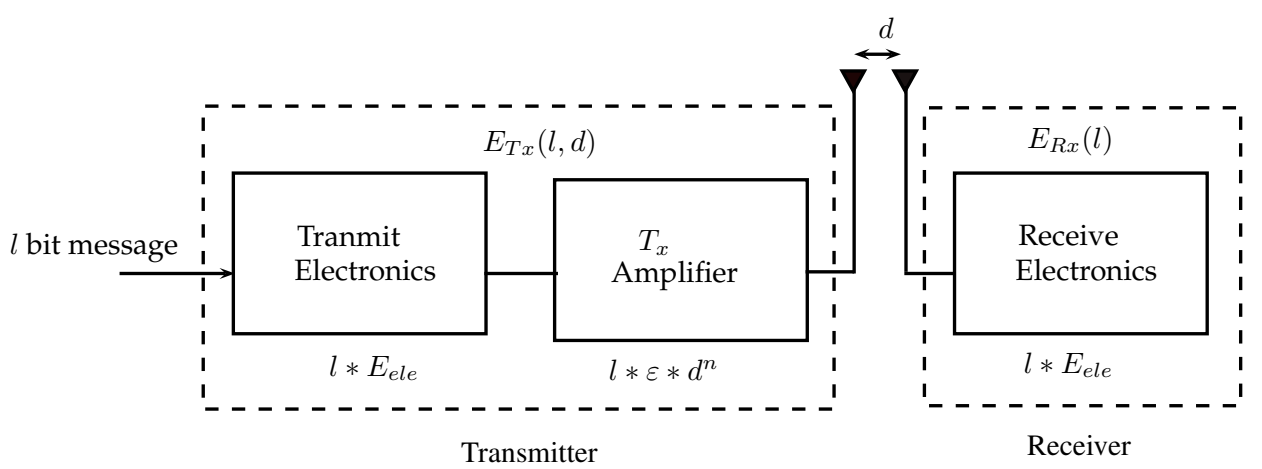

Fig. 1: Energy radio model

\subsection{Radio Model}

The radio model adopted from [6] for communication and computation energy dissipation and it is shown in Fig. 1. The multi path fading channel $\left(d^{4}\right.$ power loss model $)$ is employed whenever distance between sender and receiver is more than threshold value $d_{0}$; otherwise, the free space model $\left(d^{2}\right.$ power loss model $)$ is employed as shown in Eq. 1. Therefore, energy required to transmit $l$-bit of information over distance $d$ from sender to destination is calculated as follows

$E_{T x}(l, d)= \begin{cases}l E_{\text {ele }}+l \varepsilon_{f s} d^{2} & \text { for } d \leq d_{0} \\ l E_{\text {ele }}+l \varepsilon_{m p} d^{4} & \text { for } d>d_{0}\end{cases}$

Here, $E_{\text {ele }}$ is the energy dissipated per bit by the transmitter or receiver circuitry. The constants $\varepsilon_{f s}$ and $\varepsilon_{m p}$ are amplifier characteristics of free space channel and multi path channel respectively. The variable $d$ is the distance between transmitter and receiver; The variable $d_{0}=\sqrt{\frac{\varepsilon_{f s}}{\varepsilon_{m p}}}$, distinguishes two types of path loss model.

The amount of energy dissipated by the receiver after receiving $l$-bit data packet is calculated as follows:

$E_{R x}(l)=l E_{\text {ele }}+l E_{D A}$

Here, $E_{D A}$ is the energy dissipated by the $\mathrm{CH}$ for aggregating a one bit information.

\section{Proposed Model}

\subsection{Assumptions}

- All sensor nodes in the network are homogeneous and having same initial energy, memory, and communicating capabilities.

- Sensor nodes and base station are stationary after deployment.

- Cluster setup phase is similar to the LEACH or CROSS algorithm to select appropriate cluster heads.

- Elected cluster heads notify their residual energy, distance to base station and centrality to BS.

- After receiving information from $\mathrm{CHs}$ base station employs fuzzy logic inference system to select SCHs.

- Election of SCHs probability is decided on three input parameters residual energy, distance to base station, and centrality of CHs.

- Association of member nodes with cluster nodes and association of cluster nodes with super cluster nodes are established according to their distance metric as described in LEACH.

- The data transmission in the network happens in the steady phase of the network. Firstly, from sensor nodes $\mathrm{CHs}$ and secondly, $\mathrm{CH}$ s to $\mathrm{SCH}$, and finally from $\mathrm{SCH}$ to Base station as shown in Fig. 2.

The proposed FLAG and I-FLAG hybrid hierarchical clustering models are divided into 


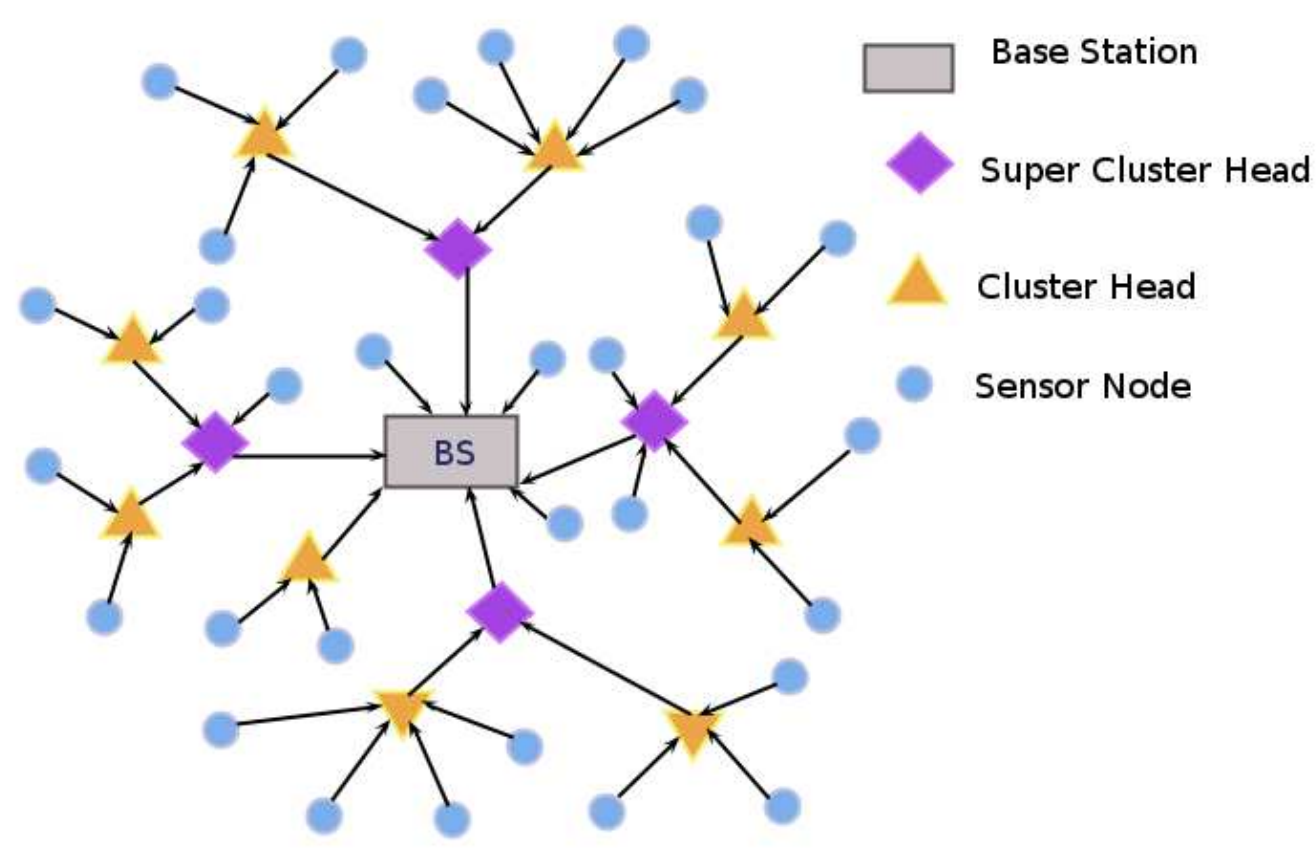

Fig. 2: An instance of network model

cluster heads selection phase and super cluster heads selection phase. Table 2 shows all the definitions of variables and acronyms that are used in this article.

\subsection{Cluster Heads Selection Phase}

In this phase, an existing CROSS and an improved version of CROSS game theoretic protocol are used.

According to the definition of game theory, clustering game(CG) of WSN with $N$ nodes, $A$ actions, and $U$ utility function are represented with three tuples as $C G(N, A, U)$. where tuples $N, A$, and $U$ denote the player set, strategy set, and payoff set of the game respectively. Suppose sensor nodes adopt pure strategy to play the clustering game, then the strategy space or action profile has two choices; either node is declared $(D)$ to be cluster head or not declared $(N D)$ to be cluster head. With regard to utility, if none of the sensor nodes select strategy $D$, then utility for each sensor node will be 0 . Sensor node payoff will be $v$, if atleast one of its neighbor sensor node is declared to be cluster head and such cluster head payoff will be $v-c$, since it plays the role of forwarding packets to the base station. Suppose there are only two players, then the Table 3 gives utility values of the two player game. Therefore, in general the utility value for any player $i$ which plays action $a_{i}$ from action profile set $A$ in a $N$ player game will take the following form 
Table 2: List of notations

\begin{tabular}{ll}
\hline Notation & Description \\
\hline$d$ & distance \\
$l$ & Data packet size in bits \\
$E_{\text {ele }}$ & Energy consumed for 1-bit computation \\
$E_{T x}(l, d)$ & Energy consumed to transmit $l$-bit data over $d$ distance \\
$E_{R x}(l)$ & Enery consumed to recieve $l$-bit data \\
$\varepsilon_{m p}$ & Amplifier characteristics of the multi path channel \\
$\varepsilon_{f s}$ & Amplifier characteristics of the free space channel \\
$\left.E_{D A}\right)$ & Energy consumed for aggregate data \\
$w_{m i n}$ & User defined variable to avoid negative value during CH probability calculation \\
$N$ & Number of sensor nodes \\
$A$ & Actions available for sensor nodes \\
$U_{i}\left(a_{i}\right)$ & Utility gained by a sensor node $i$ after choosing an action $a_{i}$ \\
$D$ & Sensor node declared to be cluster head \\
$N D$ & Sensor node not declared to be cluster head \\
$D$ & Sensor node declared to be cluster head \\
$v$ & Payoff for being cluster member \\
$c$ & Energy overhead for being cluster head \\
$R$ & Average radius of a cluster \\
$E_{c h i}$ & Energy drain of node $i$ to become CH \\
$E_{c m i}$ & Energy drain by a cluster member node $i$ to transmit data to CH \\
$D_{\text {ri }}$ & Degree of node $i$ \\
$E_{i}$ & Residual energy of a sensor node $i$ \\
$E_{o}$ & Initial energy of a sensor node \\
$d_{i}$ & Distance between the sensor node $i$ and base station \\
$d_{m a x}$ & Distance between farthest alive node to the base station \\
$B S_{\text {distance }}$ & Distance to base station \\
$R_{\text {energy }}$ & Residual energy of a CH \\
$C H$ centrality & Centrality of a CH \\
$E_{s p e n t}$ & Energy spent so far by the CH \\
$N_{b r}$ & Neighbors of CH \\
$\beta$ & Random variable takes the value between 0 and 1 \\
$Q$ & CHEF local distance \\
\hline
\end{tabular}

Table 3: Utility for two player clustering game

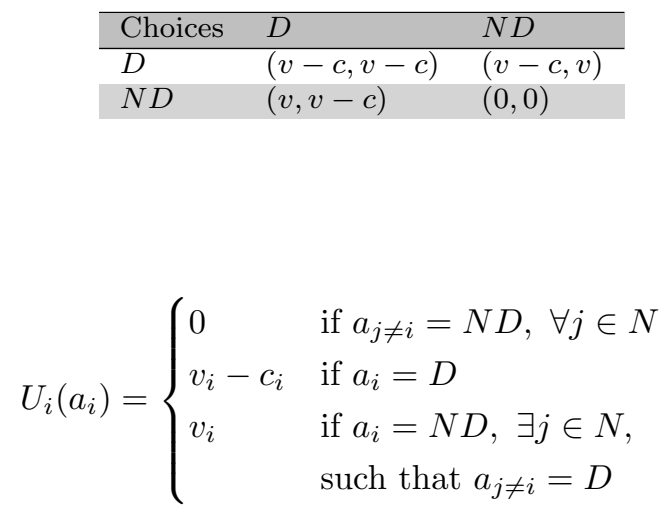

The payoff or utility value $v_{i}$ for player $i$ when it choose action $N D$, and if atleast one other player choose action $D$ can be computed as follows [32]

$$
v_{i}=\frac{l}{E c m_{i}}
$$

where $l$ is the size of the data packet and $E \mathrm{~cm}_{i}$ is the energy drain to transmit the packet to its respective $\mathrm{CH}$ [32], and can be computed as follows

$$
E c m_{i}=l E_{\text {ele }}+\frac{4}{9} l \varepsilon_{f s} R^{2}
$$

Here, $R$ is the average radius of each cluster. Suppose node $i$ chooses the action $D$, then its utility value is computed as follows [32]. 
$v_{i}-c_{i}=\frac{\left(D r_{i}+1\right) l}{E c h_{i}}$

where $c_{i}$ is the overhead of a node for becoming $\mathrm{CH}, D r_{i}$ is the node degree of a palyer $i$, and $E c h_{i}$ is the energy drain for node $i$ for becoming $\mathrm{CH}[32]$. Its value is obtained as follows

$E c h_{i}=D r_{i} l E_{\text {ele }}+\left(D r_{i}+1\right) l E_{D A}+l \varepsilon_{m p}\left(d_{i}{ }^{t o B S}\right)$

From Eq.(4) and (6), $c_{i}$ value can be calculated as follows

$c_{i}=\frac{l}{E c m_{i}}-\frac{\left(D r_{i}+1\right) l}{E c h_{i}}$

Eq.(8) takes the form by denoting $w_{i}=\frac{c_{i}}{v_{i}}$, as follows

$w_{i}=1-\frac{\left(D r_{i}+1\right) E c m_{i}}{E c h_{i}}$

To avoid the $w_{i}$ value to be negative, a parameter $w_{\min }$ is introduced, and $w_{i}$ is obtained as follows.

$w_{i}=\max \left(w_{\min }, 1-\frac{\left(D r_{i}+1\right) E c m_{i}}{E c h_{i}}\right)$

Suppose the sensor node $i$ that chooses the action $D$ with probability $p_{i}$ and the action $N D$ with probability $q_{i}=1-p_{i}$. Then, there exists an equilibrium probability $p_{i}$ based on mixed strategy nash equilibrium as discussed in CROSS protocol [9], and is formally defined as follows

$p_{i}=1-\left(w_{i}\right)^{\frac{1}{N-1}}$

Along the above equation we use residual energy and distance of the node to the base station to get appropriate probability for cluster head selection. The new improved version of CROSS for cluster head selection can be expressed as follows

$p_{i}=1-\left(w_{i}\right)^{\frac{1}{N-1}} \times\left[\left(\frac{E_{i}}{E_{o}}\right)^{\beta}+\left(\frac{d_{i}}{d_{\max }}\right)^{1-\beta}\right]$

where $\beta \in(0,1)$ is a weight factor, $E_{i}$ is the remaining energy and the $E_{o}$ is the initial energy of a node $i$ respectively. The variables $d_{i}$ and $d_{\max }$ are distance between node $i$ and BS and distance to the farthest alive node to the BS respectively .

\subsection{Super Cluster Heads Selection Phase}

In this phase, Fuzzy inference System(FIS) is employed to elect appropriate $\mathrm{SCH}$ from set of CHs.

FIS is a data base generally employed to handle uncertainties and imprecise information in the system. It consists of member functions and fuzzy rules. There are three types of FIS: Mamdani, Sugeno, and Tsukamoto. The Mamdani FIS is a popular and widely adopted technique in different applications because of its intuitive characteristic and simple structure [28].

The main comoponents of FIS are Fuzzifier, Fuzzy rules, Inference engine, and Defuzzifier as shown in Figure 3 .

In first step, crisp set inputs are transformed into fuzzy sets using membership functions, and the process is known as fuzzification. In second step, The fuzzy inference engine uses "IF-THEN" rules to map fuzzy input sets for fuzzy output sets. At last, fuzzy outputs are transfered to crisp values, and the process is called defuzzification.

Input membership function variables considered for FIS are residual energy, distance to base station, and centrality of CHs.

- Residual Energy of CH ( $\left.R_{\text {energy }}\right)$ : Selection of higher residual energy $\mathrm{CH}$ node to be $\mathrm{SCH}$ prolongs the stability of the network. 


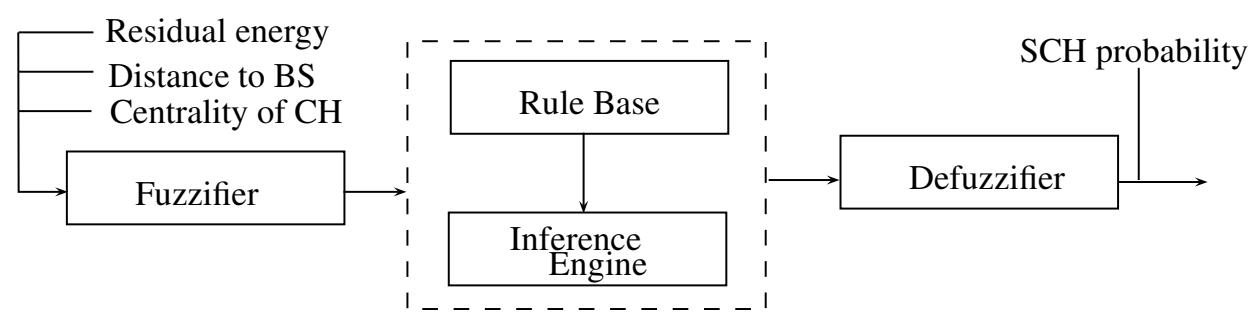

Fig. 3: Outline of fuzzy model

Table 4: Fuzzy membership functions

\begin{tabular}{lllll}
\hline SerialNo & Input variable & \multicolumn{3}{c}{ Linguistic variables } \\
\hline 1 & Residual energy & Low & Medium & High \\
2 & Distance to BS & Near & Average & Far \\
3 & Centrality of CH & Close & Reachable & Distant \\
\hline
\end{tabular}

$R_{\text {energy }}=E_{o}-E_{\text {spent }}$

- Centrality of $\mathrm{CH}\left(\mathrm{CH}_{\text {centrality }}\right)$ : This value corresponds to position of $\mathrm{CH}$ in compared to its neighbors with in the entire network. $\mathrm{CHs}$ with low centrality values have given high priority than the $\mathrm{CHs}$ with high centrality values.

$C H_{\text {centrality }}(j)=\frac{\sqrt{\frac{\sum_{i \in N b r(j)} d^{2}\left(C H_{j}, C H_{i}\right)}{|N b r(j)|}}}{M}$

where $d\left(\mathrm{CH}_{j}, \mathrm{CH}_{i}\right)$ is the distance between the $j^{\text {th }} \mathrm{CH}$ node to the $i^{\text {th }} \mathrm{CH}$ node, $M$ is the dimension of the network.

- Distance to Base Station $\left(B S_{\text {distance }}\right)$ : This value corresponds to distance between $\mathrm{CH}$ to the BS, the lower the value, lower the energy consumption. And hence $\mathrm{CHs}$ which are nearer to the BS have given higher priorities than $\mathrm{CHs}$ that are far away from the BS.

$B S_{\text {distance }}(i, j)=\sqrt{\left.\left(x_{i}-x_{j}\right)^{2}+\left(y_{i}-y_{j}\right)^{2}\right)}$
The FIS input membership functions for $\mathrm{SCH}$ selection with their linguistic variables are given in Table 4.

The membership functions for $R_{\text {energy }}$, $C H_{\text {centrality }}$, and $B S_{\text {distance }}$ are represented in Fig. 4, Fig. 5, and Fig. 6 respectively. These membership functions are mapped to "IF-THEN" rules which are mentioned in Table. 5 and Mamdani FIS is employed to handle uncertainty of selecting appropriate SCHs. There are 27 rules containing all combination of 3 input variables. The member function of the output fuzzy variable called probability having 9 combinations of linguistic values as shown in Fig.7. The trainagular member ship function is used for all input and output variables. The final value for probability is defuzzified to crisp value using centroid method. The centroid is computed as follows

Centroid $=\frac{\sum_{i} \mu\left(x_{i}\right) x_{i}}{\sum_{i} \mu\left(x_{i}\right)}$

where $\mu\left(x_{i}\right)$ is the membership value for the point $x_{i}$ in the universe of discourse. The surface view of input/output of membership functions with fuzzy rules are shown in Figures [ 8-10] 


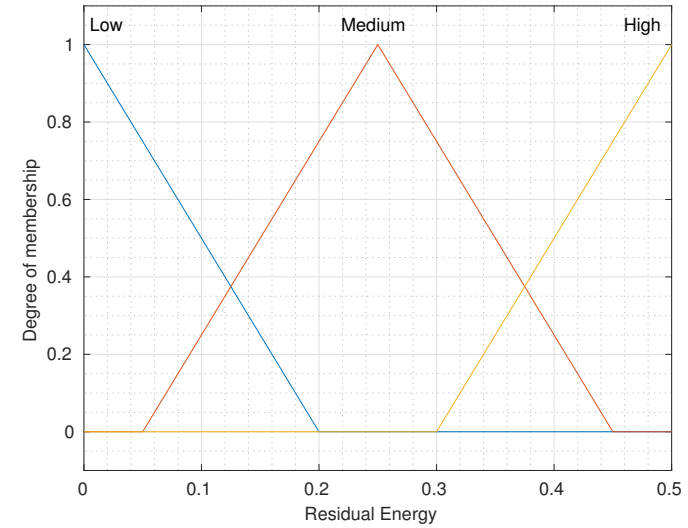

Fig. 4: Residual energy of $\mathrm{CH}$

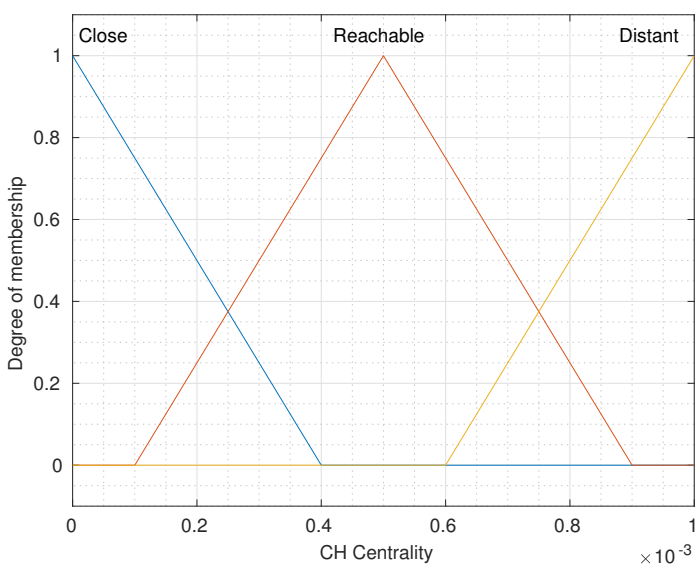

Fig. 5: Centrality of $\mathrm{CH}$

The complete flow of the proposed scheme is depicted in Algorithm 1.

\section{Results and Discussion}

Experiments are carried out using MATLAB 2019a software to evaluate and compare the performance of proposed protocols with the existing protocols like LEACH [2], CHEF [8], and CROSS [9]. Experiments are repeated many times, and the average results are taken to handle the randomness of simulations. To conduct experiments 100 sensor nodes are randomly placed on $100 \mathrm{~m} \times 100 \mathrm{~m}$ field with the base station location at coordinate $(50,50)$. All sensors are homogeneous and the initial energy of each sensor is set to 0.5Joule. The sensed data size by each sensor is identical and equal to 4000 bits. The energy consumption for transmitter(receiver) electronic is set to $50 \mathrm{~nJ} /$ bit. The energy drain for data fusion is set to $5 \mathrm{~nJ} /$ bit/message. All simulation parameters used in experiments are mentioned in Table 7.

Table 7: Simulation parameters

\begin{tabular}{ll}
\hline Parameters & Values \\
\hline Number of nodes $n$ & 100 \\
Network size $\left(m^{2}\right)$ & $100 \times 100$ \\
Initial node energy $(\mathrm{J})$ & 0.5 \\
Data packet size $l$ (bits) & 4000 \\
$E_{\text {ele }}(\mathrm{nJ} / \mathrm{bit})$ & 50 \\
$E_{T x}(\mathrm{~nJ} / \mathrm{bit})$ & 50 \\
$E_{R x}(\mathrm{~nJ} / \mathrm{bit})$ & 50 \\
$\varepsilon_{m p}\left(\mathrm{pJ} / \mathrm{bit} / \mathrm{m}^{4}\right)$ & 0.0013 \\
$\varepsilon_{f s}\left(\mathrm{pJ} / \mathrm{bit} / m^{2}\right)$ & 10 \\
$E_{D A}(\mathrm{~nJ} / \mathrm{bit} / \mathrm{message})$ & 5 \\
$w_{m i n}$ & 0.000001 \\
$\mathrm{LEACH} p($ percentage $)$ & 0.05 \\
$\mathrm{CHEF}$ average cluster size $(\mathrm{m})$ & 25 \\
$\mathrm{BS}$ location(coordinates $)$ & $(50,50)$ \\
$R(\mathrm{~m})$ & 10 \\
\hline
\end{tabular}

Two hybrid clustering protocol for WSN is designed by augmenting fuzzy logic to basic CROSS and improved-CROSS to increase the stability of the network. Fig. 11 shows lifetime of the network. It is observed that the first node dies faster in other protocols compared to I-FLAG, Moreover the network stability period is better in proposed protocols until $10 \%$ of nodes die in the network. Network stability period, stability period throughput, and network lifetime are tabulated in Table 6 .

The network lifetime of I-FLAG is better than LEACH, CHEF, and I-CROSS, however it underperforms over CROSS and CROSS based FLAG because of its even energy drain distribution among nodes of the network. And 


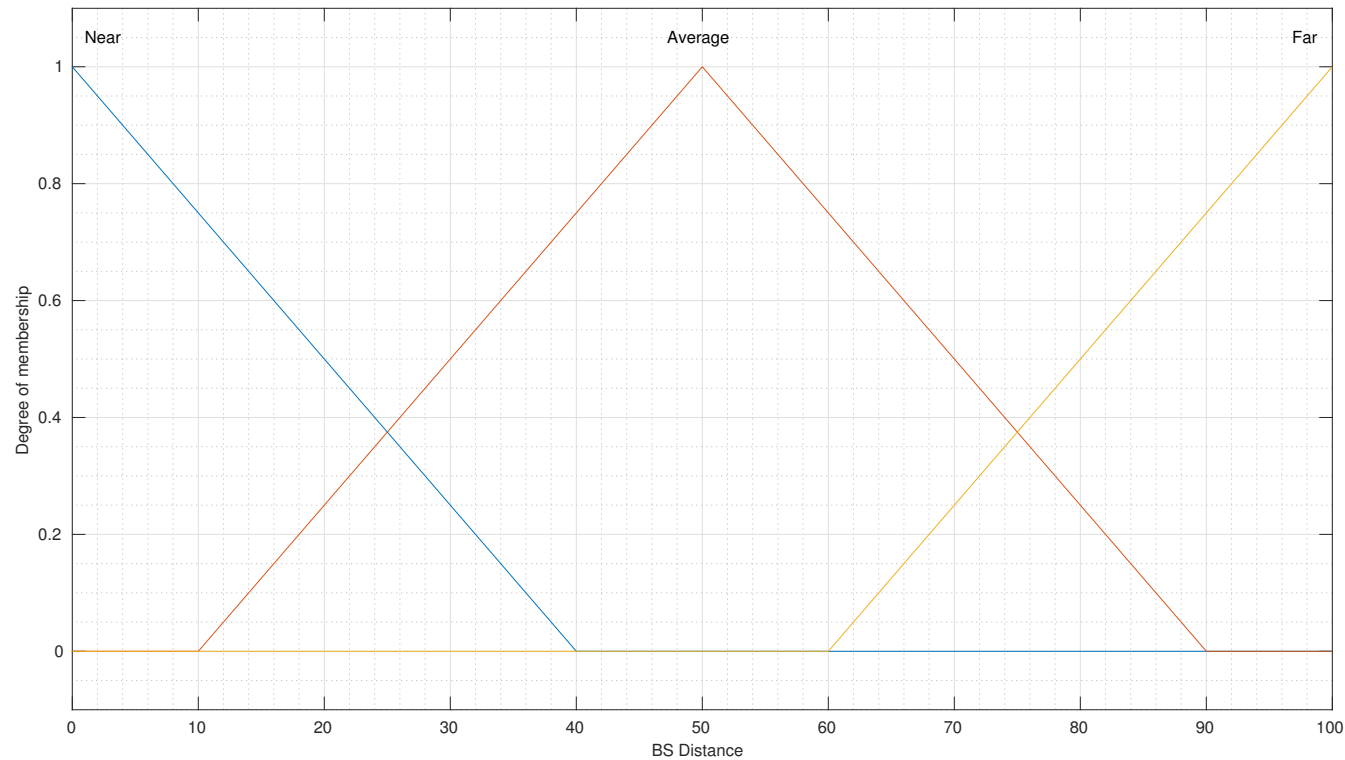

Fig. 6: Distance between $\mathrm{CH}$ to BS

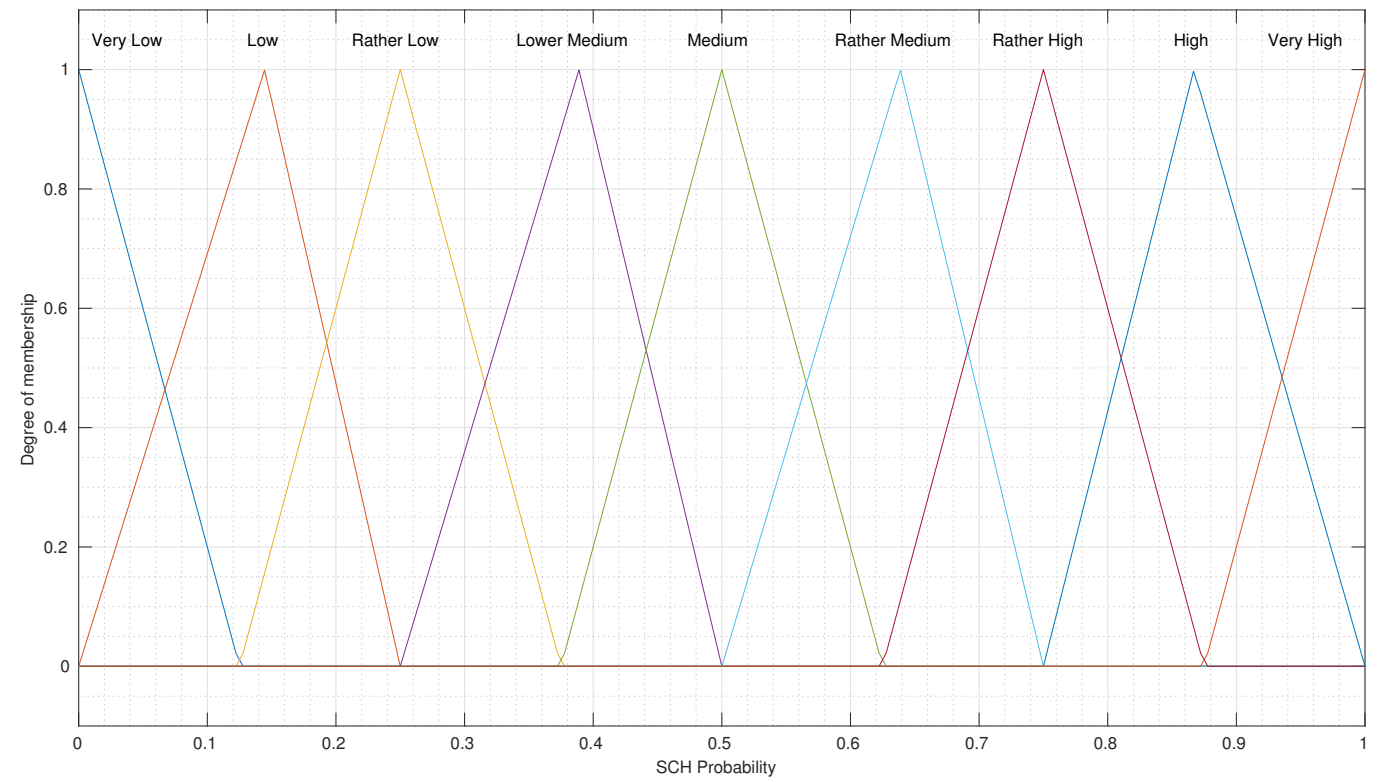

Fig. 7: SCH selection probability 
Table 5: Fuzzy rules

\begin{tabular}{lllll}
\hline RuleNo & Renergy & BS $_{\text {distane }}$ & CH $_{\text {centrality }}$ & SCH $_{\text {probability }}$ \\
\hline 1 & Low(0) & Near(0) & Close(0) & Rather Low(2) \\
2 & Low(0) & Near(0) & Reachable(1) & Low(1) \\
3 & Low(0) & Near(0) & Distant(2) & Very Low(0) \\
4 & Low(0) & Average(1) & Close(0) & Rather Low(2) \\
5 & Low(0) & Average(1) & Reachable(1) & Low(1) \\
6 & Low(0) & Average(1) & Distant(2) & Very Low(0) \\
7 & Low(0) & Far(2) & Close(0) & Rather Low(2) \\
8 & Low(0) & Far(2) & Reachable(1) & Low(1) \\
9 & Low(0) & Far(2) & Distant(2) & Very Low(0) \\
10 & Medium(1) & Near(0) & Close(0) & Rather Medium(5) \\
11 & Medium(1) & Near(0) & Reachable(1) & Medium(4) \\
12 & Medium(1) & Near(0) & Distant(2) & Lower Medium(3) \\
13 & Medium(1) & Average(1) & Close(0) & Rather Medium(5) \\
14 & Medium(1) & Average(1) & Reachable(1) & Medium(4) \\
15 & Medium(1) & Average(1) & Distant(2) & Lower Medium(3) \\
16 & Medium(1) & Far(2) & Close(0) & Rather Medium(5) \\
17 & Medium(1) & Far(2) & Reachable(1) & Medium(4) \\
18 & Medium(1) & Far(2) & Distant(2) & Lower Medium(3) \\
19 & High(2) & Near(0) & Close(0) & Very High(8) \\
20 & High(2) & Near(0) & Reachable(1) & High(7) \\
21 & High(2) & Near(0) & Distant(2) & Rather High(6) \\
22 & High(2) & Average(1) & Close(0) & Very High(8) \\
23 & High(2) & Average(1) & Reachable(1) & High(7) \\
24 & High(2) & Average(1) & Distant(2) & Rather High(6) \\
25 & High(2) & Far(2) & Close(0) & Very High(8) \\
26 & High(2) & Far(2) & Reachable (1) & High(7) \\
27 & High(2) & Far(2) & Distant(2) & Rather High(6) \\
\hline & & & & \\
\hline
\end{tabular}

Fig. 8: Surface view of Distance to BS, Residual energy, and Probability

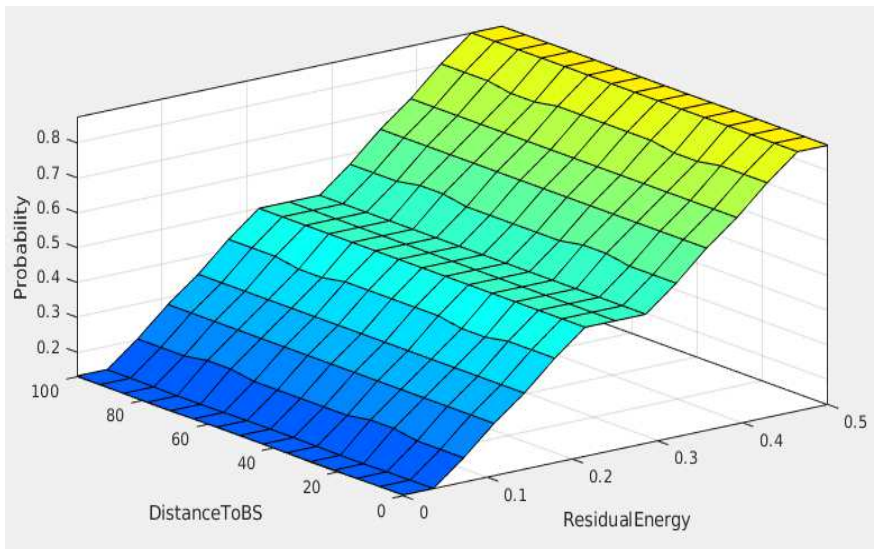

hence it prolongs the death of the first node in the I-FLAG. Fig. 12 depicts the average energy drain of the network, and it is smooth in the proposed protocols compared to other protocols. Fig. 13 and Fig. 14 represents the number of alive nodes and dead nodes of the network respectively. In the initial period of the network, number of alive nodes are more, and the number of dead nodes are less in both FLAG and I-FLAG over other protocols. The network stability period comparison is shown in Fig. 15. The graph demonstrates that the stability period of FLAG is $23 \%, 13 \%$, and $0.4 \%$ better than LEACH, CHEF, and CROSS respectively. Similarly, the stability period of I-FLAG is $25 \%, 15 \%$, and $1.2 \%$ better than 
Fig. 9: Surface view of Residual energy, Node centrality, and Probability

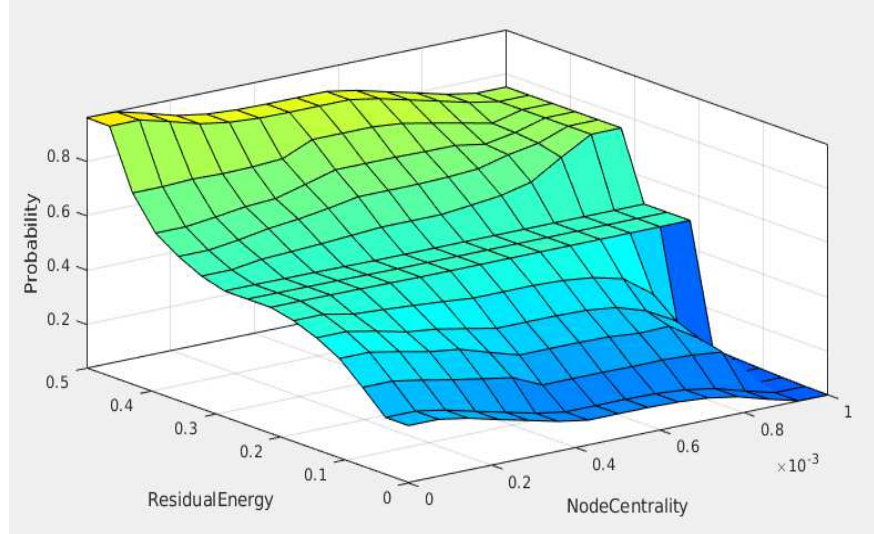

Fig. 10: Surface view of Node centrality, Distance to BS, and Probability
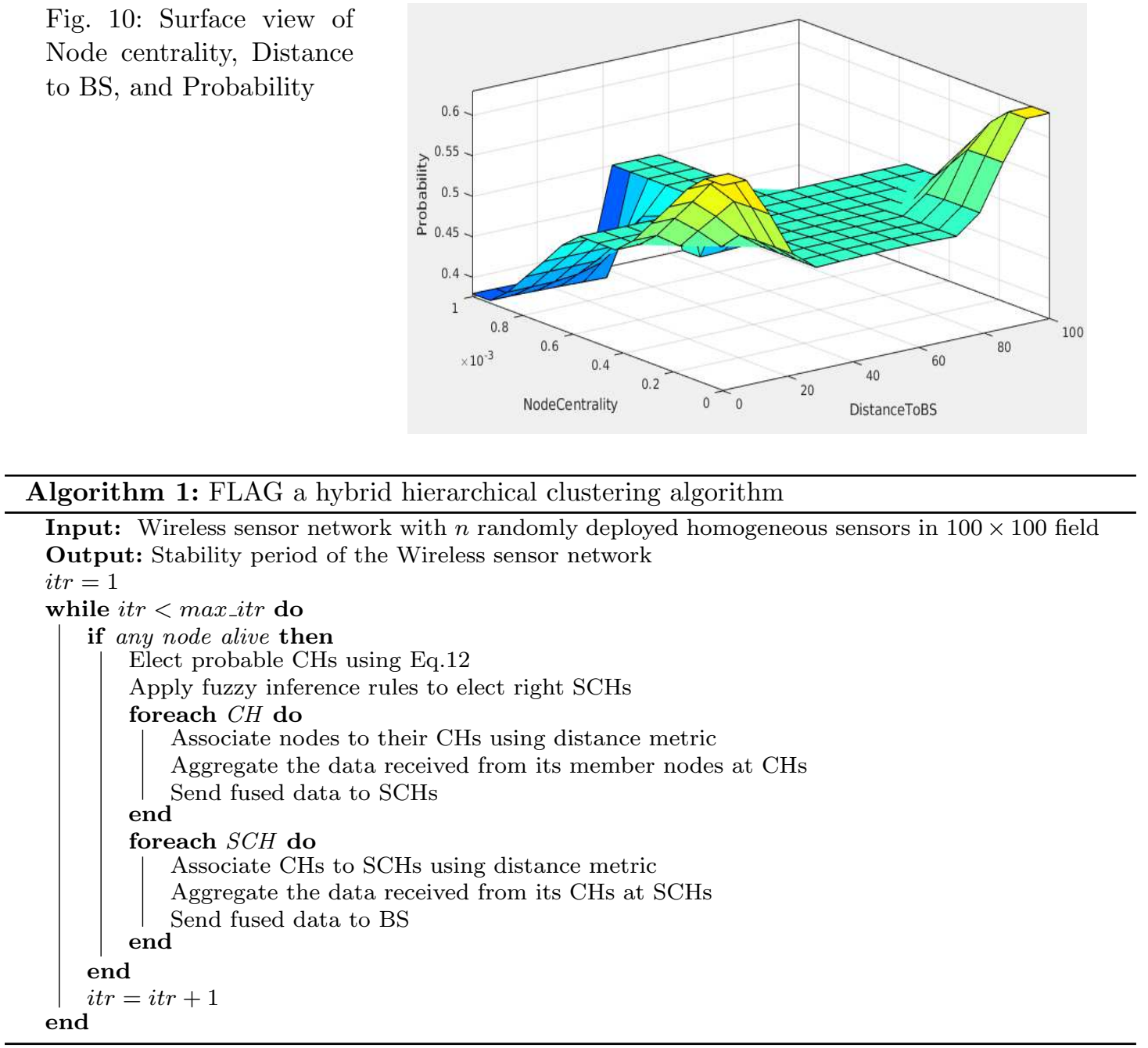
Table 6: Stability period throughput and total network lifetime

\begin{tabular}{lllllll}
\hline Parameters & LEACH & CHEF & CROSS & I-CROSS & FLAG & I-FLAG \\
\hline Stability period(rounds) & 1109 & 1206 & 1358 & 1368 & 1364 & 1386 \\
Stability period throughput(bits) & 110900 & 120600 & 135800 & 136800 & 136582 & 137572 \\
Network lifetime(rounds) & 1636 & 1373 & 2118 & 1906 & 2111 & 2051 \\
\hline
\end{tabular}

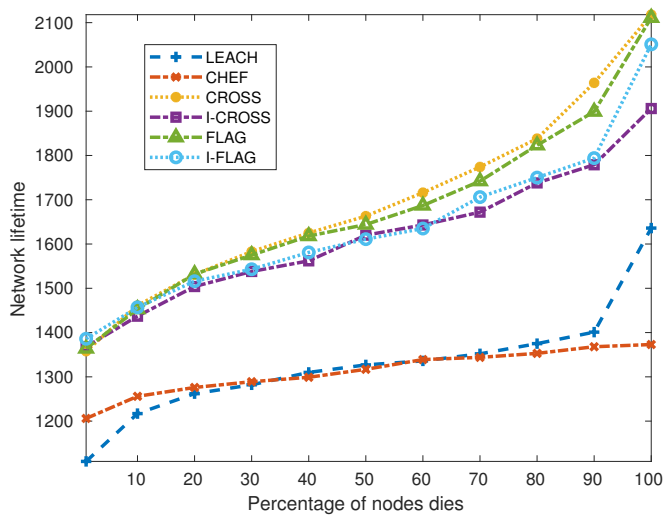

Fig. 11: Percentage of node dies Vs Network lifetime

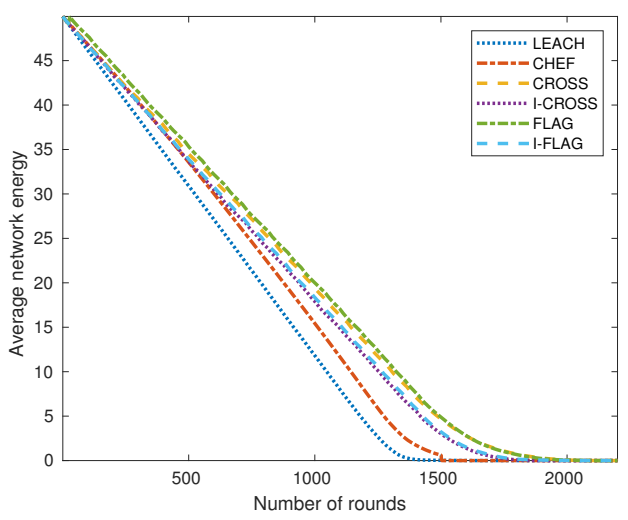

Fig. 12: Number of rounds Vs Average network energy

LEACH, CHEF, and I-CROSS respectively.The network throughput during stability period is depicted in Fig. 16. It is observed that the throughput of FLAG during the stability period is $23 \%, 13 \%$, and $0.6 \%$ better than $\mathrm{LEACH}$,

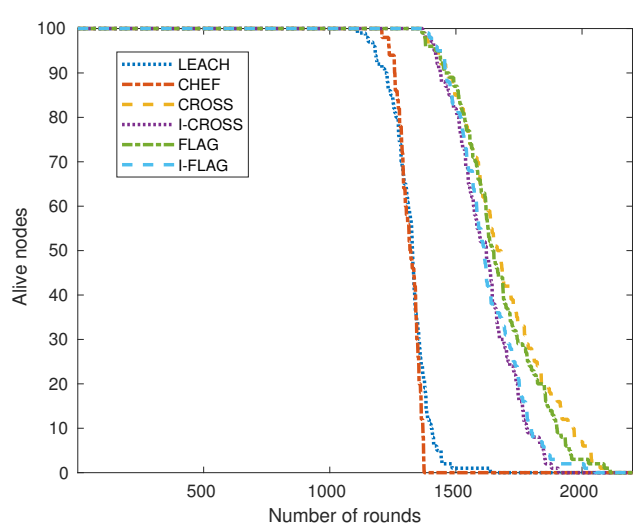

Fig. 13: Number of rounds Vs Alive nodes

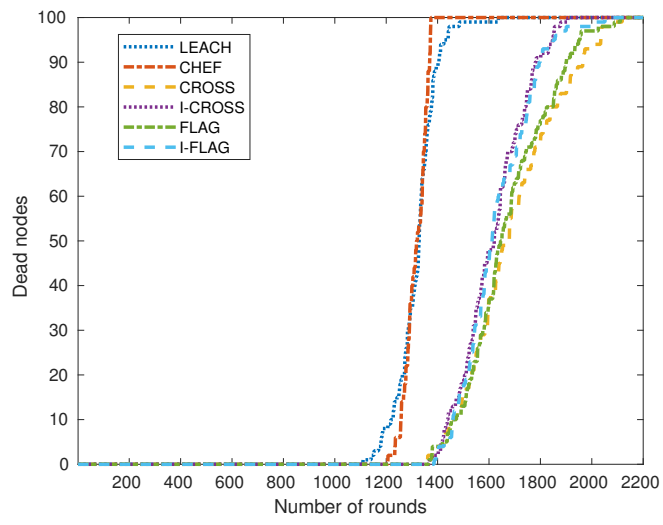

Fig. 14: Number of rounds Vs Dead nodes

CHEF, and CROSS respectively. Similarly, the stability period throughput of I-FLAG is $24 \%$, $14 \%$, and $0.5 \%$ better than LEACH, CHEF, and I-CROSS respectively.

The energy parameter and distance parameter plays a vital role in selecting $\mathrm{CH}$ and hence the SCH. It infers that the network stability 


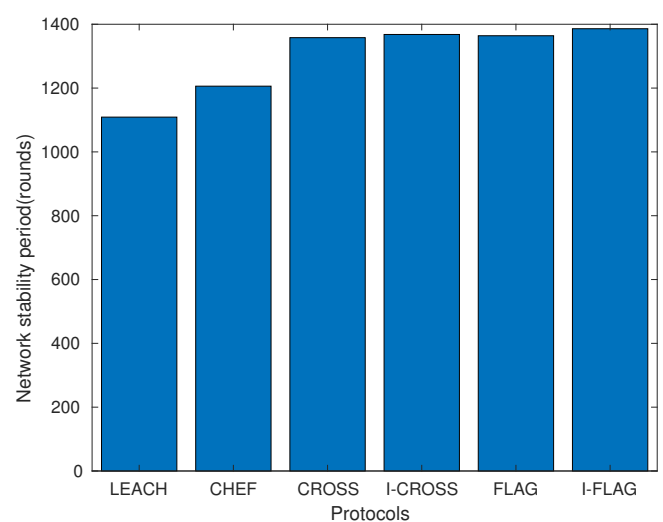

Fig. 15: Protocols Vs Network stability period

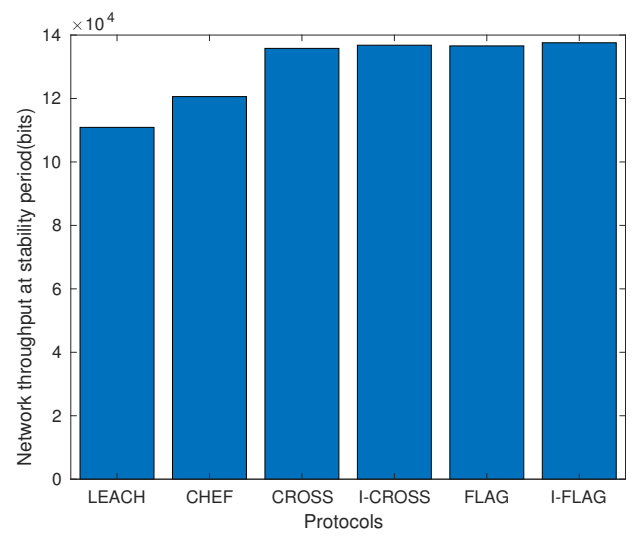

Fig. 16: Protocols Vs Network throughput at stability period

period of I-FLAG is outperforms over all other protocols.

\section{Conclusion}

Hybrid hierarchical protocols named FLAG and I-FLAG are proposed in the paper to extend network stability. Two main phases of these protocols are $\mathrm{CH}$ election phase and $\mathrm{SCH}$ election phase. The $\mathrm{CH}$ election phase is achieved through a game-theoretic protocol, and $\mathrm{SCH}$ election phase is achieved through a fuzzy inference system. The simulations are carried out to verify the superiority of the proposed pro- tocols. The results show that the network stability period of FLAG is better than LEACH, CHEF, and CROSS. The network stability period of I-FLAG is better than LEACH, CHEF, and I-CROSS. Due to the balanced energy drain of the proposed work, the network stability is better over the other protocols.

\section{Declaration}

\subsection{Funding}

Not applicable

\subsection{Conflicts of interest}

Authors declares that they does not have any conflict of interest.

\subsection{Availability of data and material}

Not applicable

\subsection{Code availability}

Not applicable

\subsection{Authors' contributions}

The authors proposed a hybrid hierarchical artificial intelligence based clustering techniques, named FLAG and I-FLAG. The first phase of these algorithms use game-theoretic technique to elect suitable cluster heads $(\mathrm{CHs})$ and later phase of the algorithms use fuzzy interference system to select appropriate super cluster heads(SCHs) among CHs. The I-FLAG is an improved version of FLAG, where additional parameters like energy and distance are considered to elect CHs. Simulations are performed to check the superiority of proposed algorithms over existing protocols. Simulation results show that the average stability period of WSN is better in FLAG and I-FLAG as compared to other protocols. 


\section{References}

1. Agrawal, D., Pandey, S.: Fuca: Fuzzy-based unequal clustering algorithm to prolong the lifetime of wireless sensor networks. International Journal of Communication Systems 31(2), e3448 (2018)

2. Akyildiz, I.F., Su, W., Sankarasubramaniam, Y., Cayirci, E.: Wireless sensor networks: a survey. Computer networks 38(4), 393-422 (2002)

3. Baranidharan, B., Santhi, B.: Ducf: Distributed load balancing unequal clustering in wireless sensor networks using fuzzy approach. Applied Soft Computing 40, 495-506 (2016)

4. Gupta, G.P., Saha, B.: Load balanced clustering scheme using hybrid metaheuristic technique for mobile sink based wireless sensor networks. Journal of Ambient Intelligence and $\mathrm{Hu}-$ manized Computing (2020)

5. Heinzelman, W.B., Chandrakasan, A.P., Balakrishnan, H.: An application-specific protocol architecture for wireless microsensor networks. IEEE Transactions on wireless communications 1(4), 660-670 (2002)

6. Heinzelman, W.R., Chandrakasan, A., Balakrishnan, H.: Energy-efficient communication protocol for wireless microsensor networks. In: Proceedings of the 33rd annual Hawaii international conference on system sciences, pp. 10-pp. IEEE (2000)

7. Jha, S.K., Eyong, E.M.: An energy optimization in wireless sensor networks by using genetic algorithm. Telecommunication Systems 67(1), 113-121 (2018)

8. Kim, J.M., Park, S.H., Han, Y.J., Chung, T.M.: Chef: cluster head election mechanism using fuzzy logic in wireless sensor networks. In: 2008 10th International Conference on Advanced Communication Technology, vol. 1, pp. 654-659. IEEE (2008)

9. Koltsidas, G., Pavlidou, F.N.: A game theoretical approach to clustering of ad-hoc and sensor networks. Telecommunication Systems 47(1-2), 81-93 (2011)

10. Kuila, P., Jana, P.K.: A novel differential evolution based clustering algorithm for wireless sensor networks. Applied soft computing 25, 414-425 (2014)

11. Lalwani, P., Banka, H., Kumar, C.: Bera: a biogeography-based energy saving routing architecture for wireless sensor networks. Soft Computing 22(5), 1651-1667 (2018)

12. Liu, Q., Liu, M.: Energy-efficient clustering algorithm based on game theory for wireless sensor networks. International Journal of Distributed Sensor Networks 13(11), 1550147717743701 (2017)

13. Logambigai, R., Kannan, A.: Fuzzy logic based unequal clustering for wireless sensor networks. Wireless Networks 22(3), 945-957 (2016)
14. Mazumdar, N., Om, H.: Distributed fuzzy logic based energy-aware and coverage preserving unequal clustering algorithm for wireless sensor networks. International Journal of Communication Systems 30(13), e3283 (2017)

15. Mazumdar, N., Om, H.: Distributed fuzzy approach to unequal clustering and routing algorithm for wireless sensor networks. International journal of Communication systems 31(12), e3709 (2018)

16. Mittal, N., Singh, U., Salgotra, R., Bansal, M.: An energy-efficient stable clustering approach using fuzzy-enhanced flower pollination algorithm for wsns. Neural Computing and Applications 32(11), 7399-7419 (2020)

17. Nayak, P., Devulapalli, A.: A fuzzy logic-based clustering algorithm for wsn to extend the network lifetime. IEEE sensors journal 16(1), 137144 (2015)

18. Neamatollahi, P., Naghibzadeh, M.: Distributed unequal clustering algorithm in largescale wireless sensor networks using fuzzy logic. The Journal of Supercomputing 74(6), 23292352 (2018)

19. Nomosudro, P., Mehra, J., Naik, C., Shetty D, P.: Ecabbo: Energy-efficient clustering algorithm based on biogeography optimization for wireless sensor networks. In: 2019 IEEE Region 10 Conference (TENCON), pp. 826-832. IEEE (2019)

20. Panchal, A., Singh, R.K.: Ehcr-fcm: Energy efficient hierarchical clustering and routing using fuzzy c-means for wireless sensor networks. Telecommunication Systems 76(2), 251-263 (2021)

21. Phoemphon, S., So-In, C., Aimtongkham, P., Nguyen, T.G.: An energy-efficient fuzzy-based scheme for unequal multihop clustering in wireless sensor networks. Journal of Ambient Intelligence and Humanized Computing (2020)

22. Premananda, B., Neti, N.K., Ashwin, K., Basu, S.V.: An energy-efficient, coalition game theory based hierarchical routing protocol for wsns. International Journal of Computers and Applications pp. 1-11 (2018)

23. Qing, L., Zhu, Q., Wang, M.: Design of a distributed energy-efficient clustering algorithm for heterogeneous wireless sensor networks. Computer communications 29(12), 2230-2237 (2006)

24. Rajaram, V., Kumaratharan, N.: Multi-hop optimized routing algorithm and load balanced fuzzy clustering in wireless sensor networks. Journal of Ambient Intelligence and Humanized Computing pp. 1-9 (2020)

25. Sindhuja, P., Ramamoorthy, P.: An improved fuzzy enabled optimal multipath routing for wireless sensor network. Cluster Computing pp. $1-9(2019)$ 
26. Smaragdakis, G., Matta, I., Bestavros, A.: Sep: A stable election protocol for clustered heterogeneous wireless sensor networks. Tech. rep., Boston University Computer Science Department (2004)

27. Thandapani, P., Arunachalam, M., Sundarraj, D.: An energy-efficient clustering and multipath routing for mobile wireless sensor network using game theory. International Journal of Communication Systems 33(7), e4336 (2020)

28. Toloueiashtian, M., Motameni, H.: A new clustering approach in wireless sensor networks using fuzzy system. The Journal of Supercomputing 74(2), 717-737 (2018)

29. Van, N.T., Huynh, T.T., An, B.: An energy efficient protocol based on fuzzy logic to extend network lifetime and increase transmission efficiency in wireless sensor networks. Journal of Intelligent \& Fuzzy Systems 35(6), 5845-5852 (2018)

30. Verma, A., Kumar, S., Gautam, P.R., Rashid, T., Kumar, A.: Fuzzy logic based effective clustering of homogeneous wireless sensor networks for mobile sink. IEEE Sensors Journal 20(10), 5615-5623 (2020)

31. Xie, D., Sun, Q., Zhou, Q., Qiu, Y., Yuan, X.: An efficient clustering protocol for wireless sensor networks based on localized game theoretical approach. International Journal of Distributed Sensor Networks 9(8), 476313 (2013)

32. Yang, L., Lu, Y.Z., Zhong, Y.C., Wu, X.G. Xing, S.J.: A hybrid, game theory based, and distributed clustering protocol for wireless sensor networks. Wireless Networks 22(3), 1007$1021(2016)$

33. Younis, O., Fahmy, S.: Heed: a hybrid, energyefficient, distributed clustering approach for ad hoc sensor networks. IEEE Transactions on mobile computing 3(4), 366-379 (2004) 
Figures

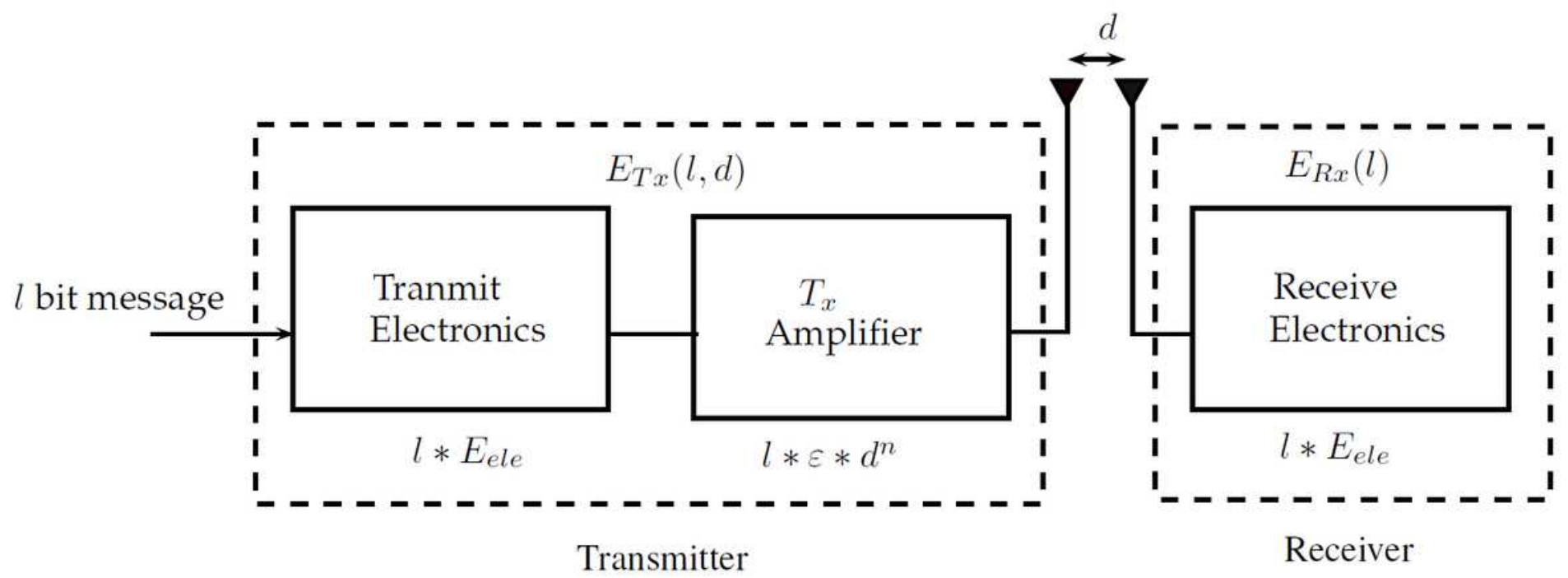

Figure 1

Energy radio model

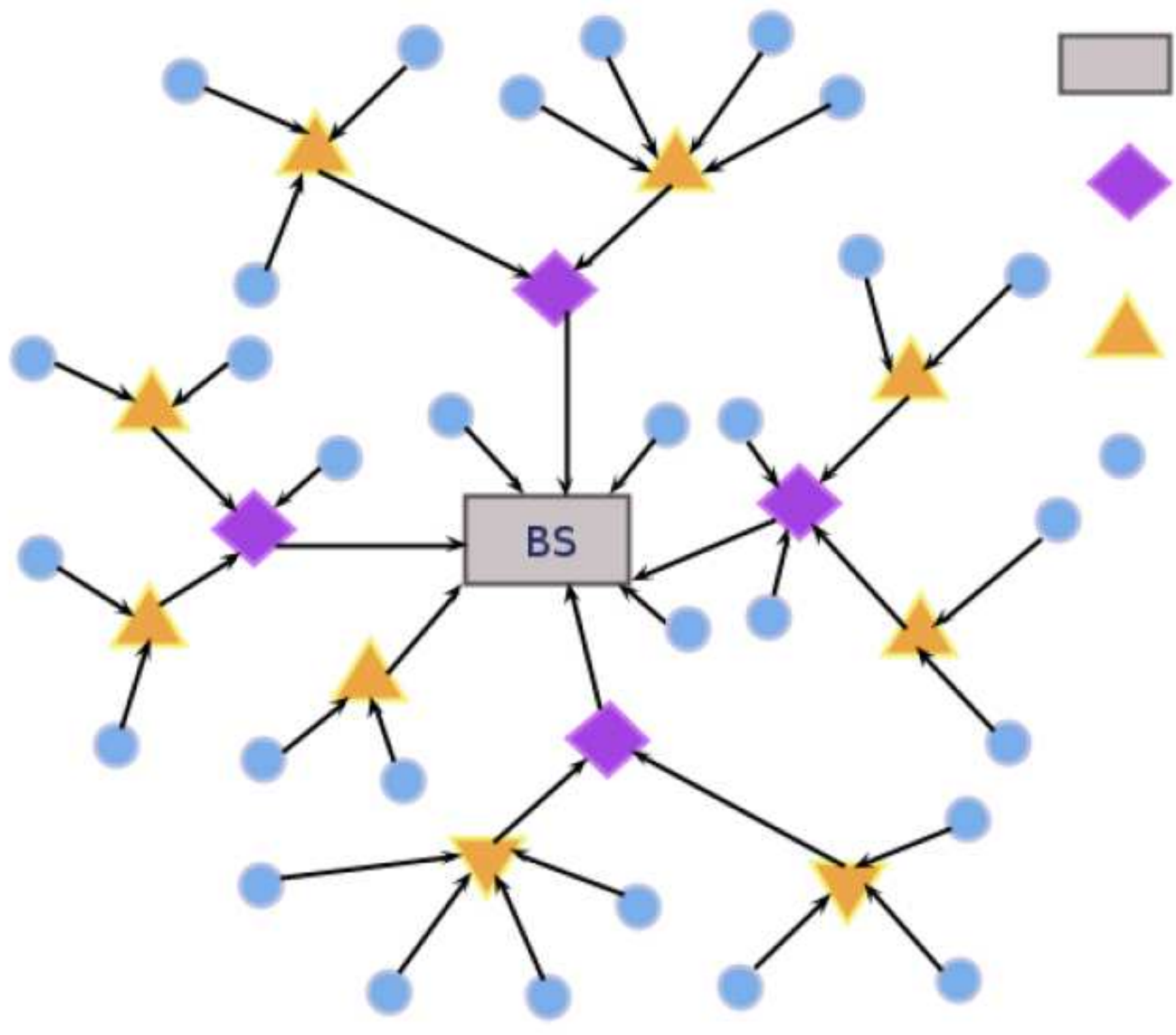

Base Station

Super Cluster Head

Cluster Head

Sensor Node

Figure 2 
An instance of network model

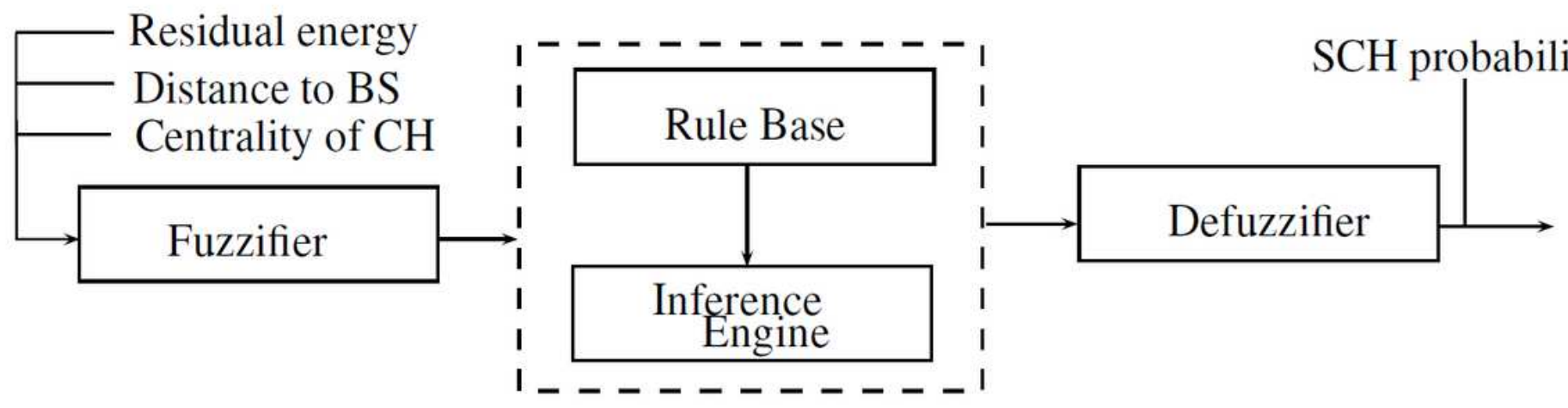

Figure 3

Outline of fuzzy model

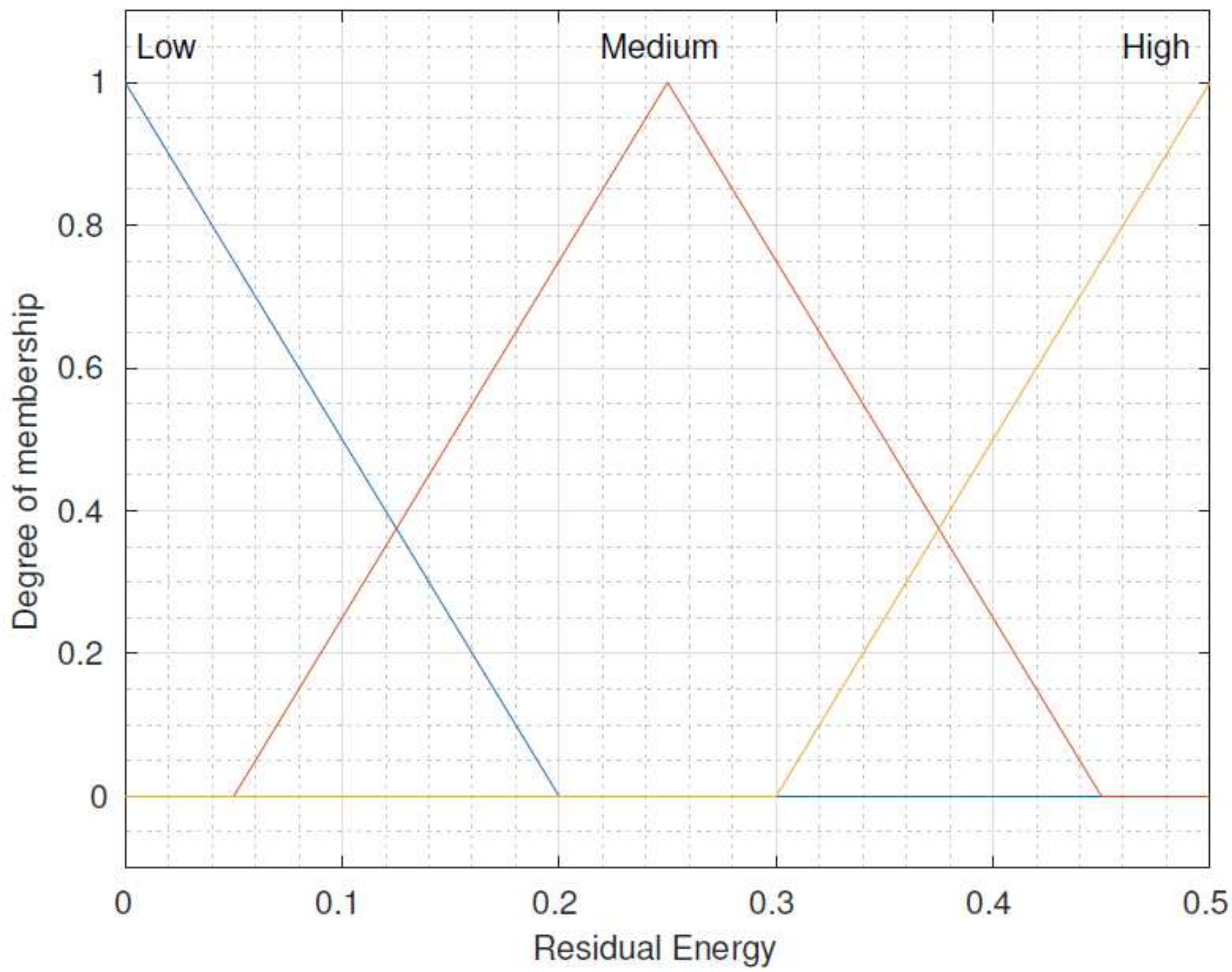

Figure 4 
Residual energy of $\mathrm{CH}$

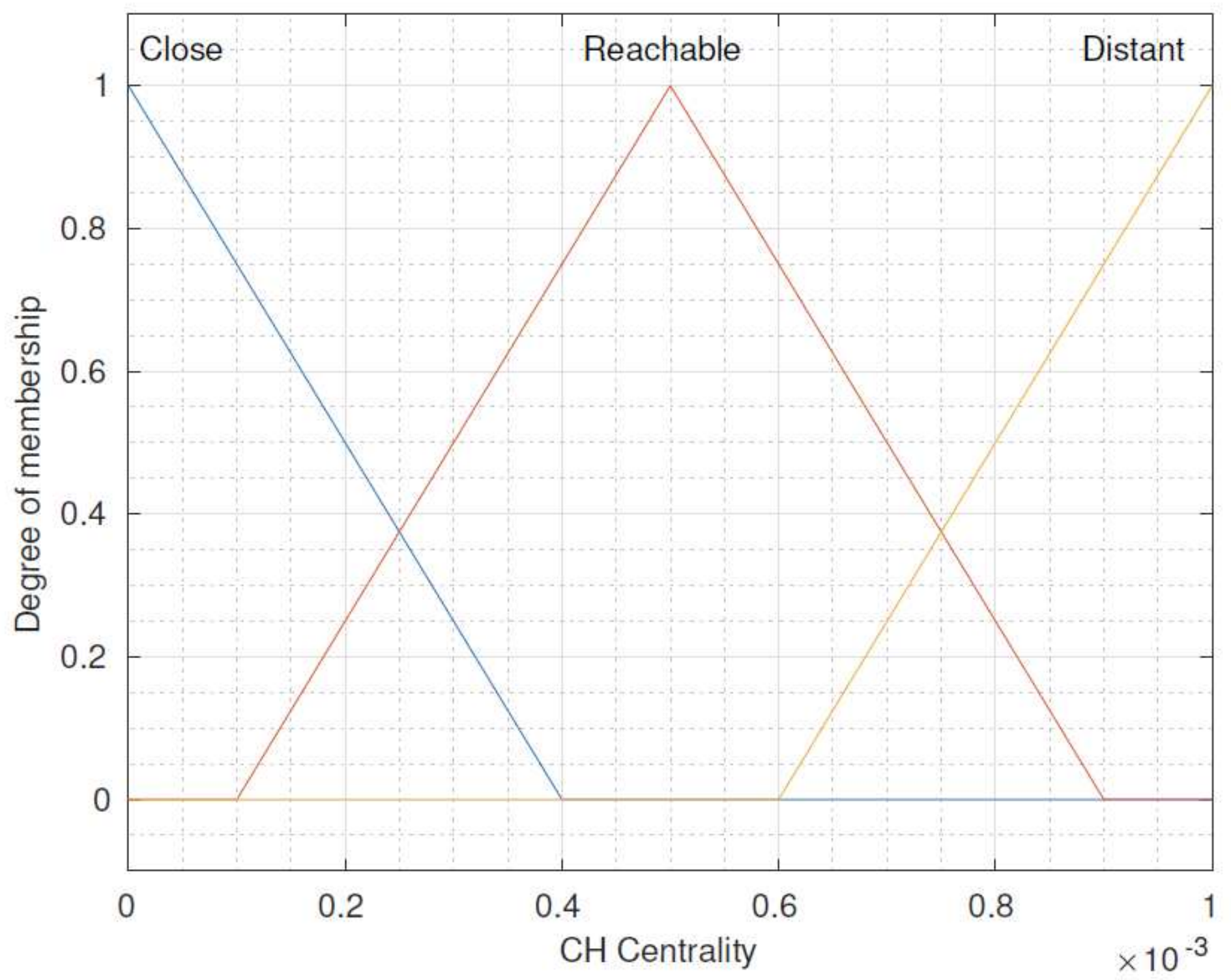

Figure 5

Centrality of $\mathrm{CH}$ 


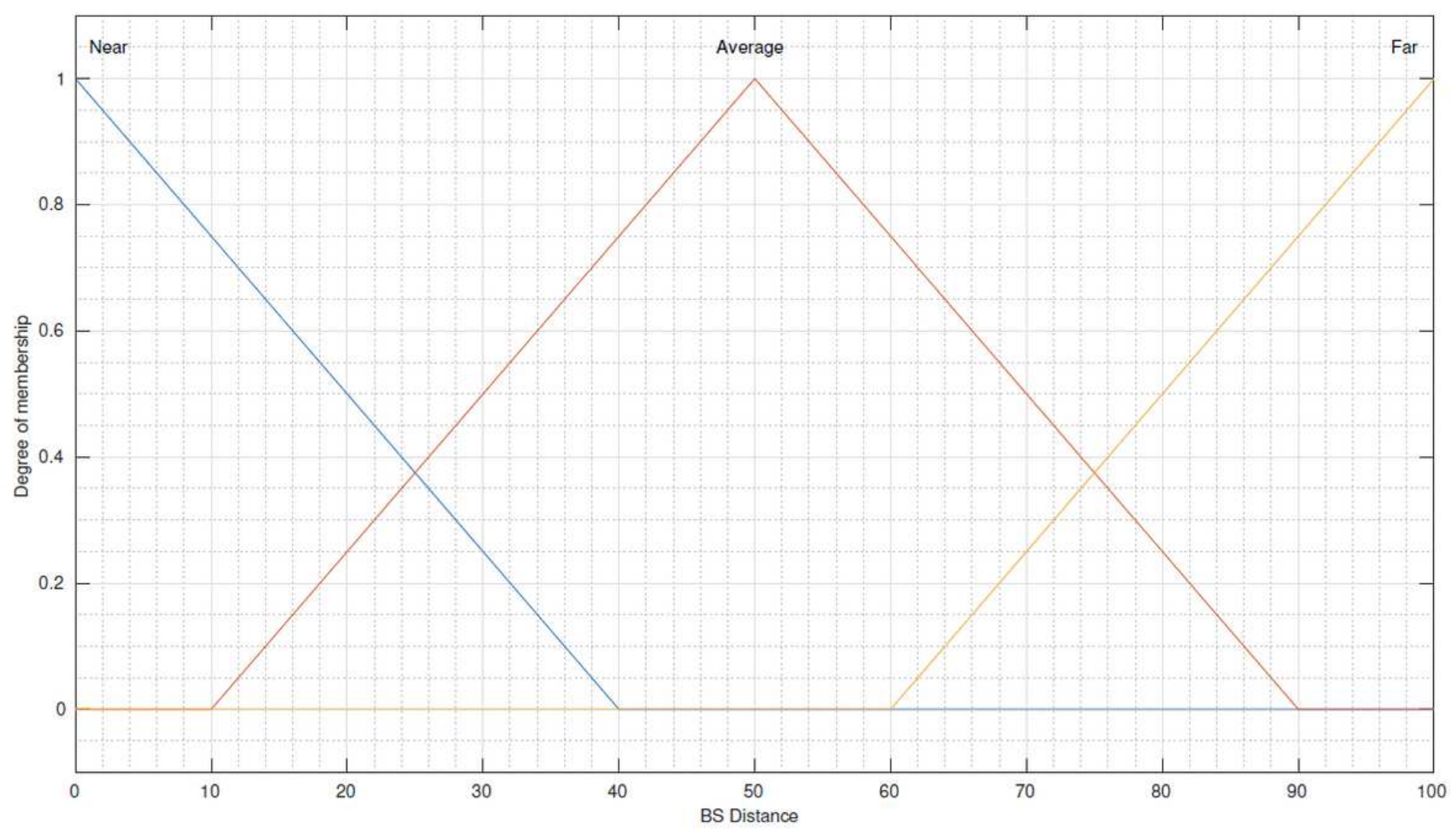

Figure 6

Distance between $\mathrm{CH}$ to $\mathrm{BS}$ 


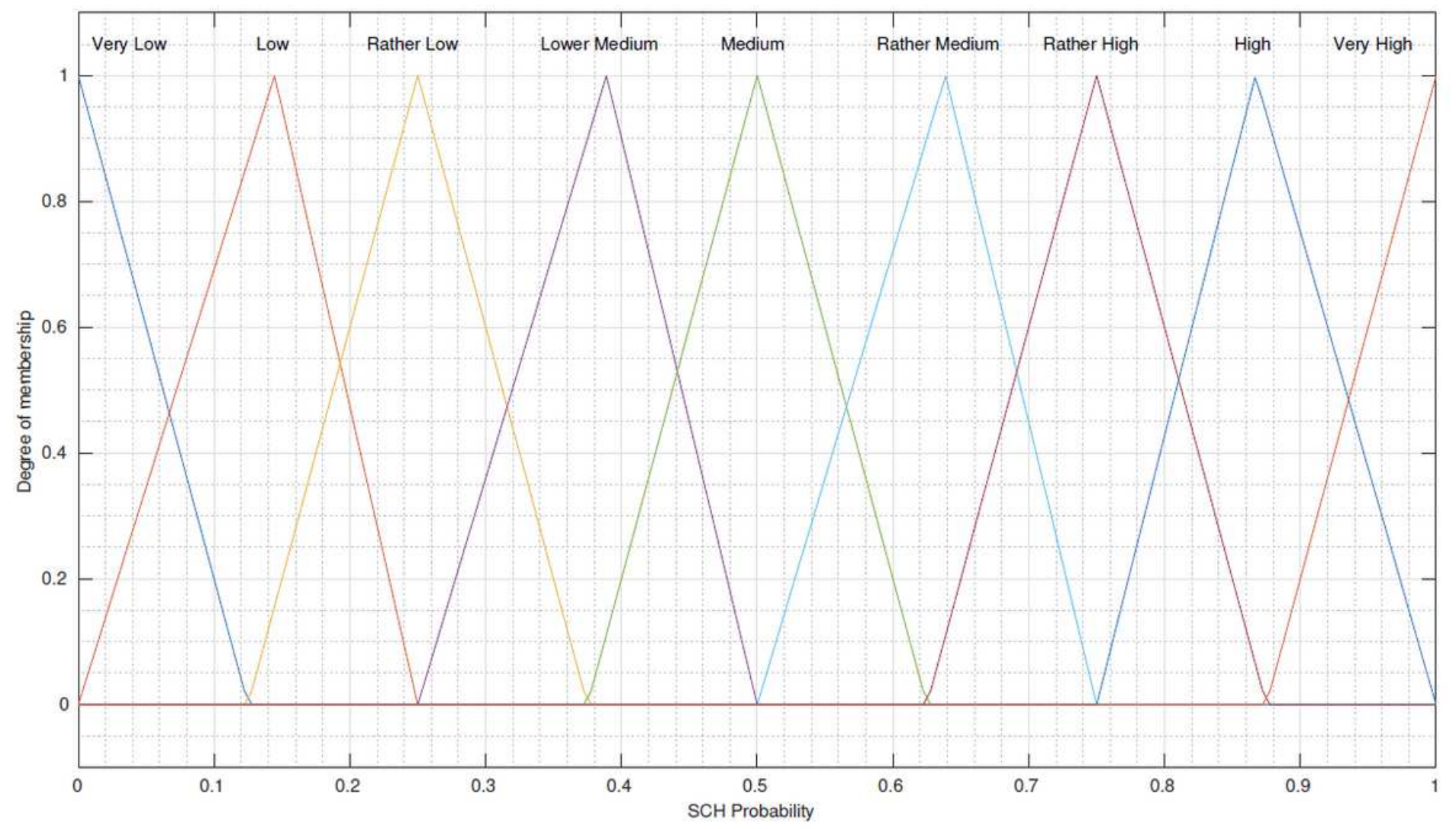

Figure 7

SCH selection probability 


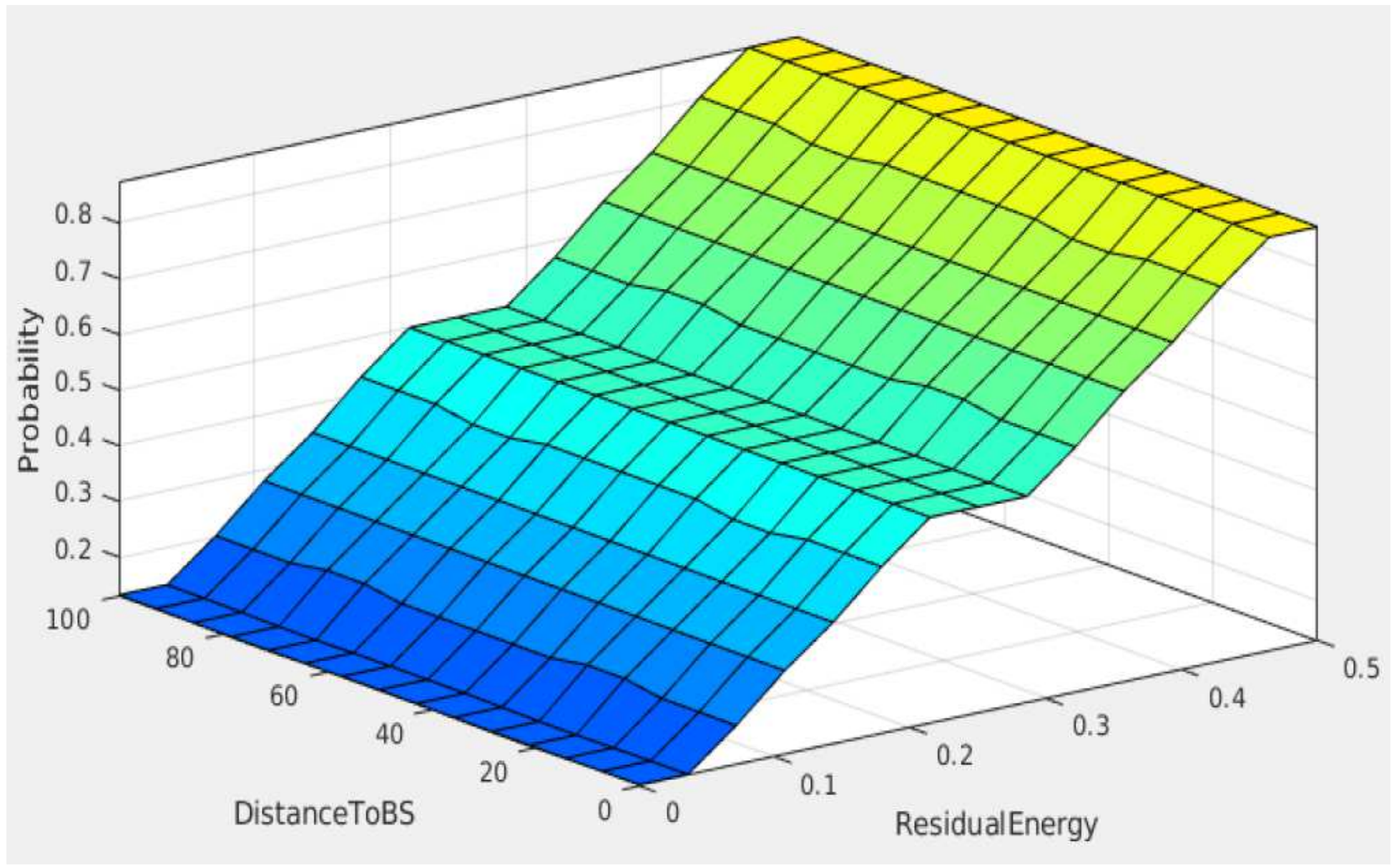

Figure 8

Surface view of Distance to BS, Residual energy, and Probability 


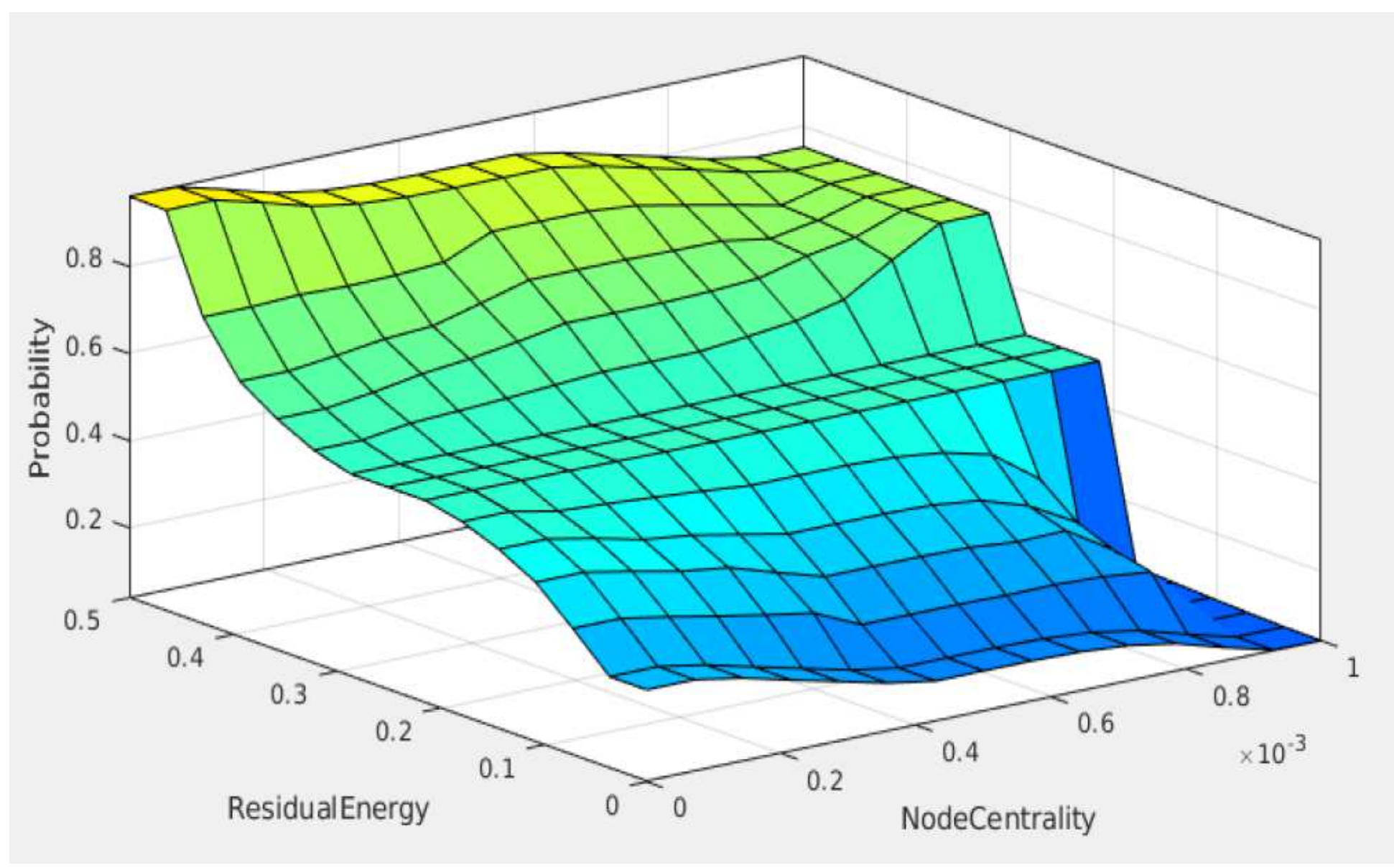

\section{Figure 9}

Surface view of Residual energy, Node centrality, and Probability 


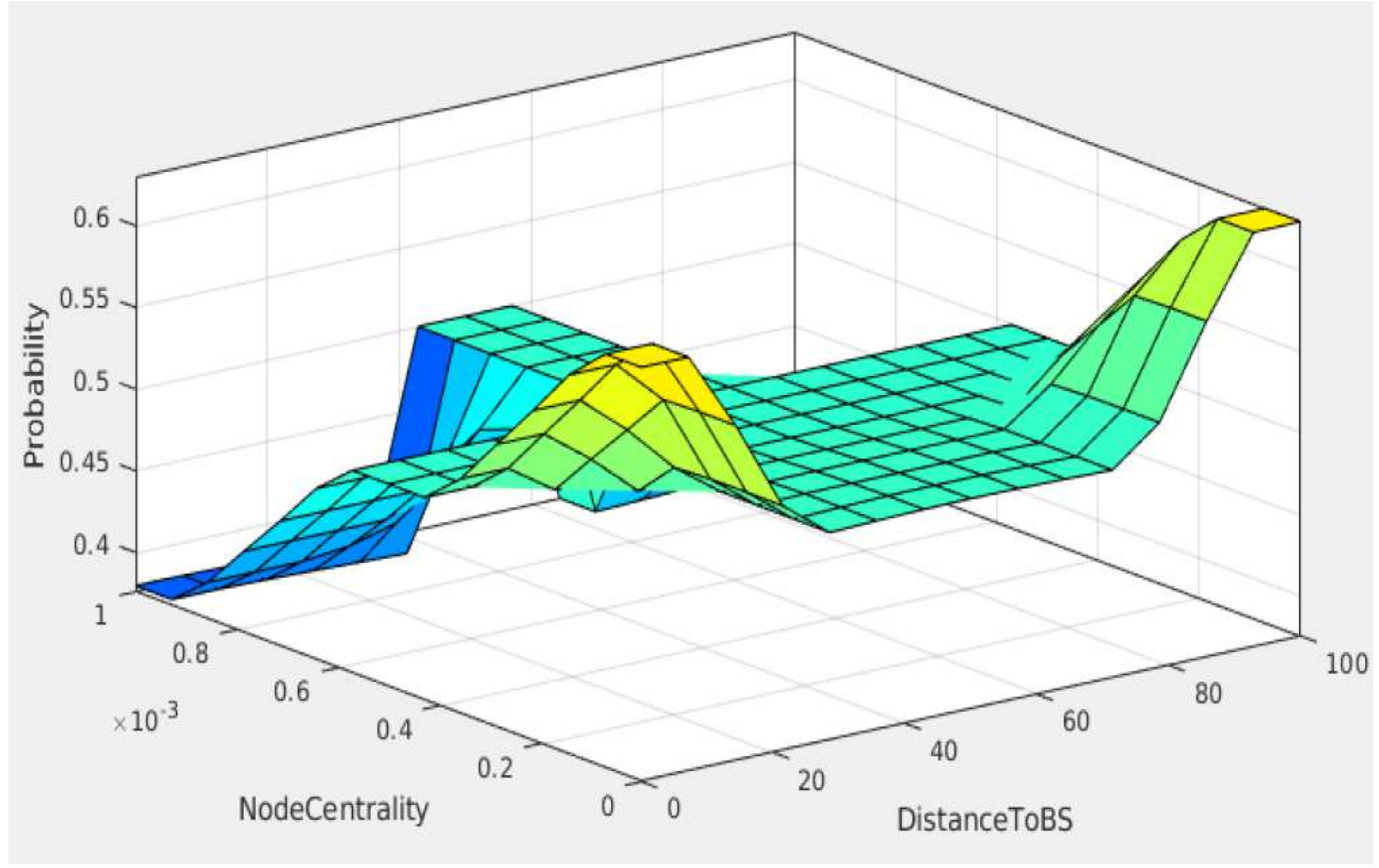

Figure 10

Surface view of Node centrality, Distance to BS, and Probability 


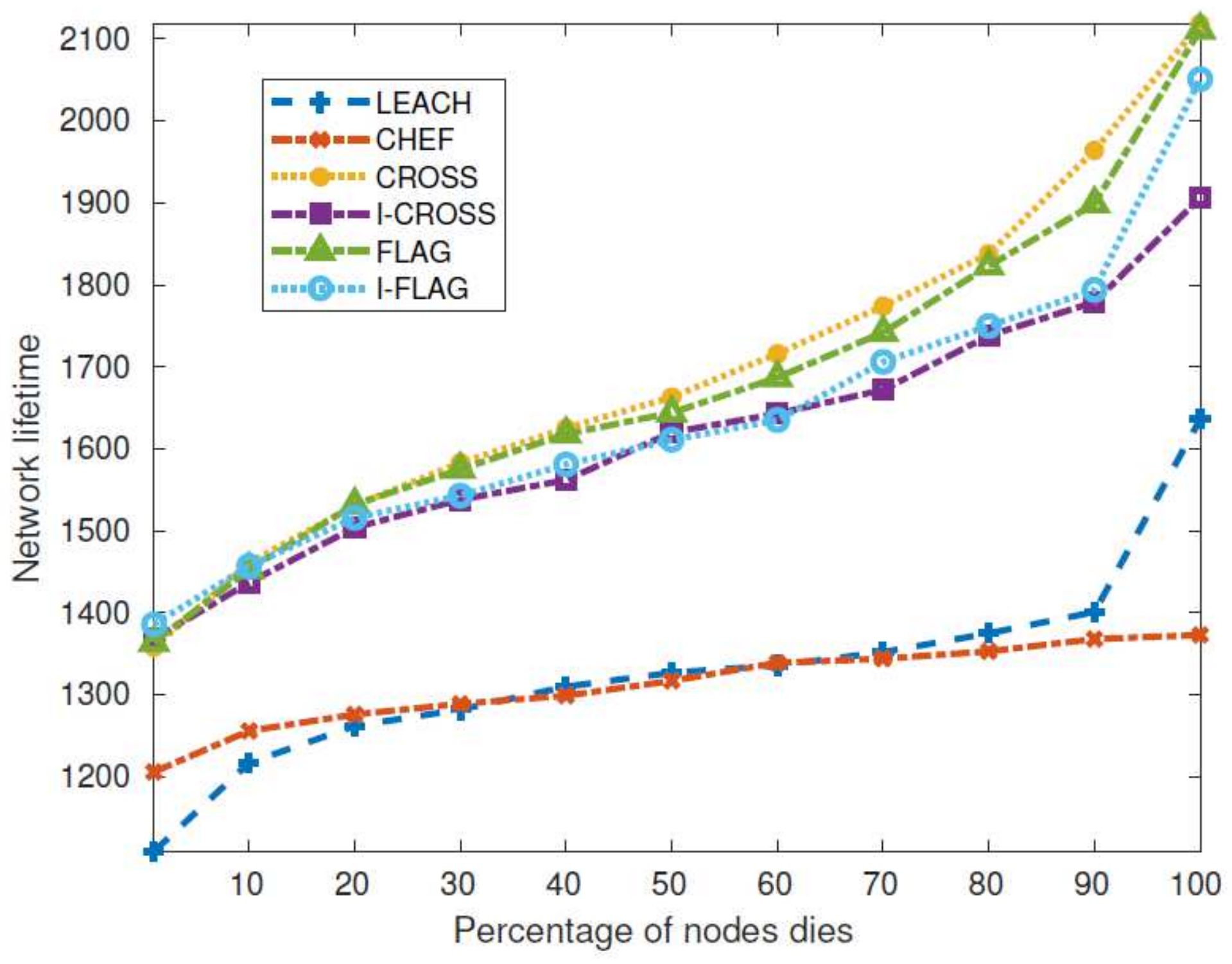

Figure 11

Percentage of node dies Vs Network lifetime 


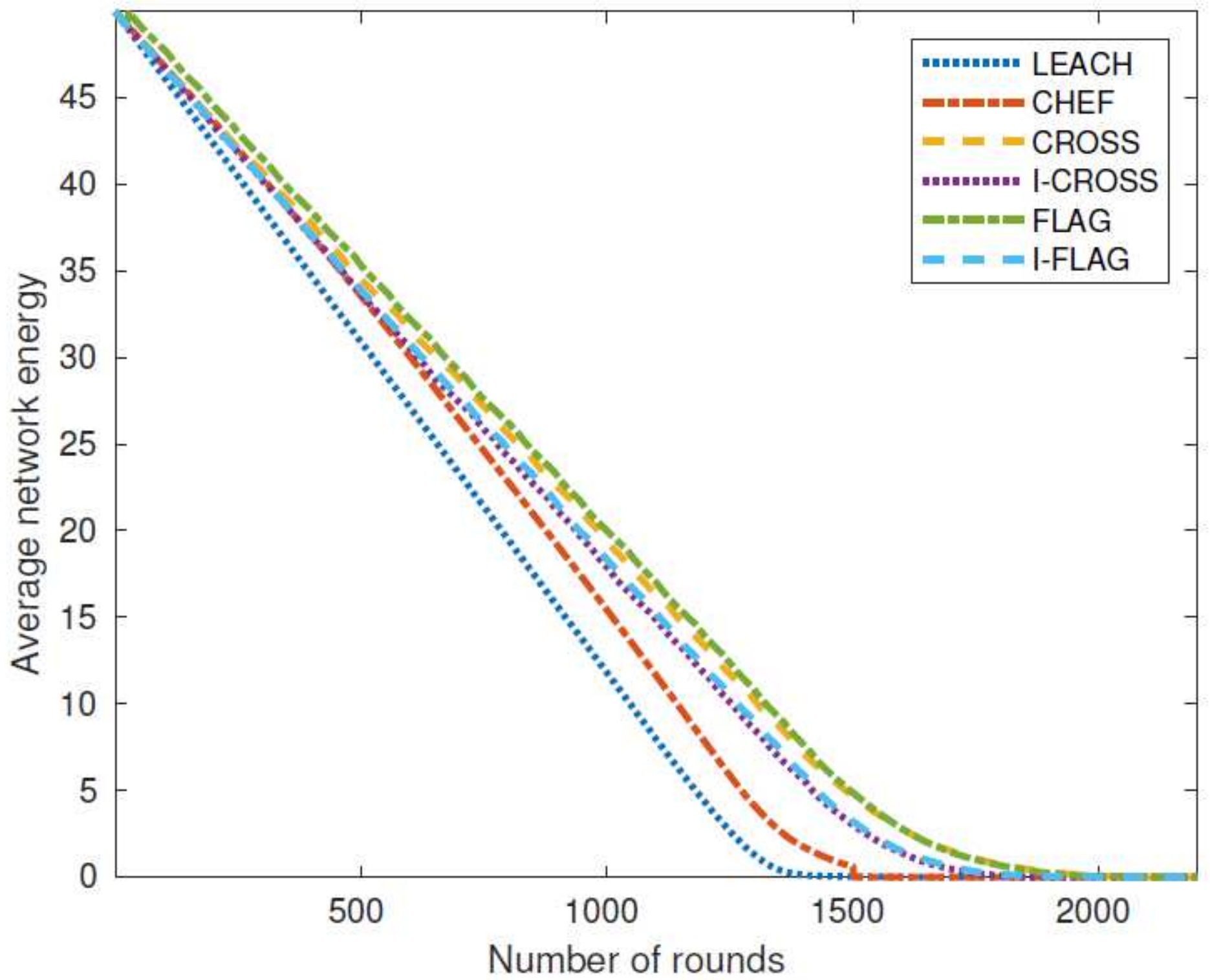

Figure 12

Number of rounds Vs Average network energy 


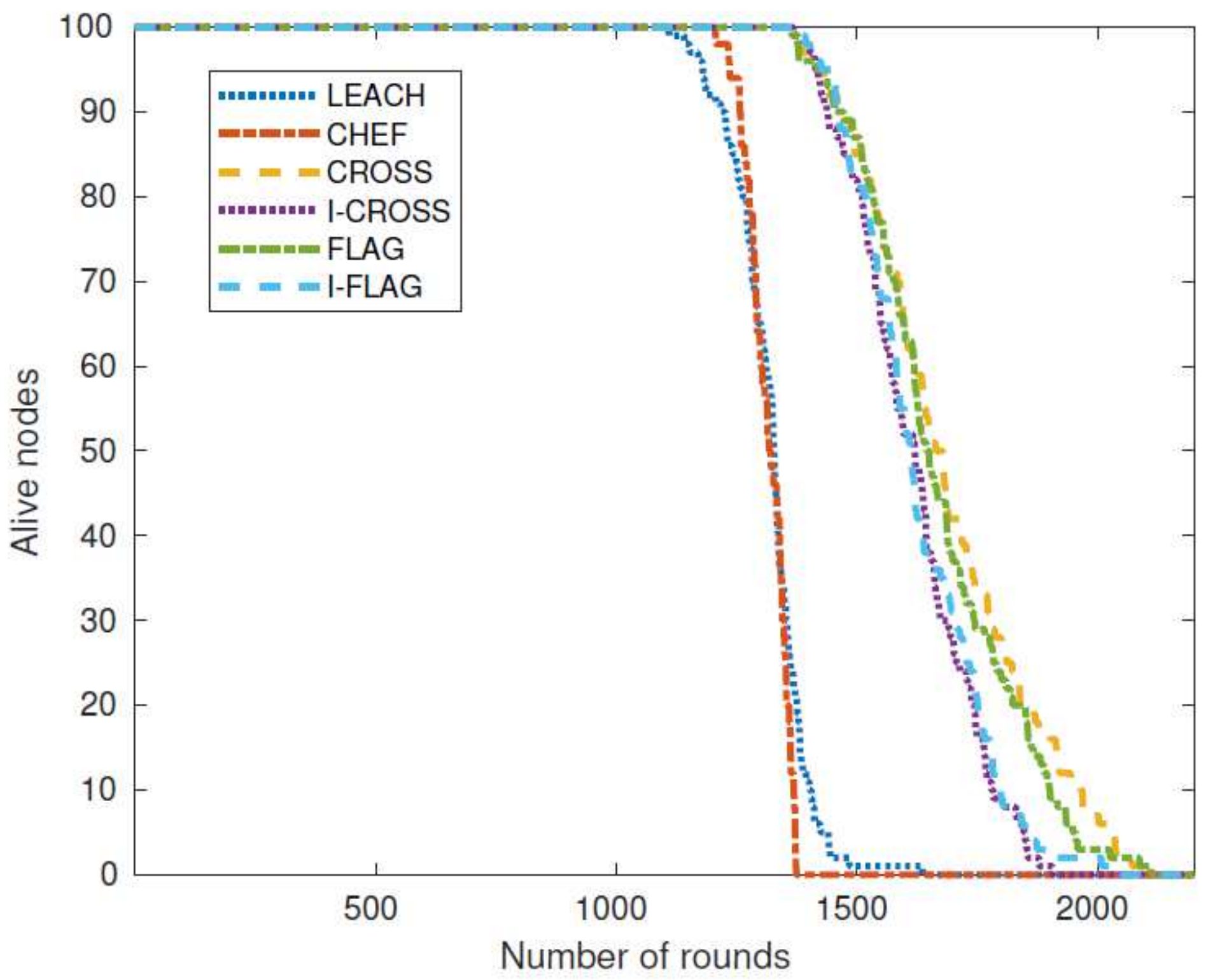

Figure 13

Number of rounds Vs Alive nodes 


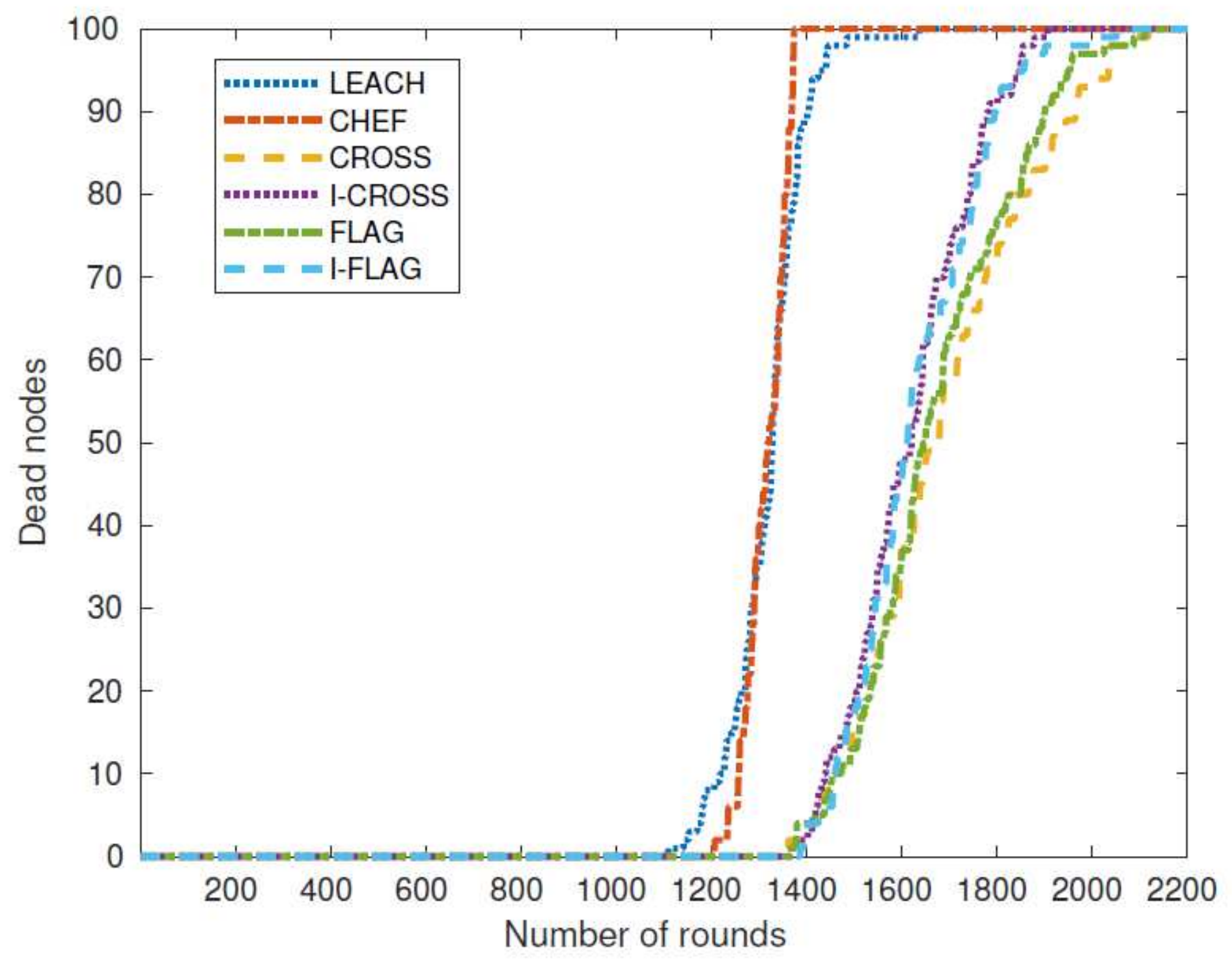

Figure 14

Number of rounds Vs Dead nodes 


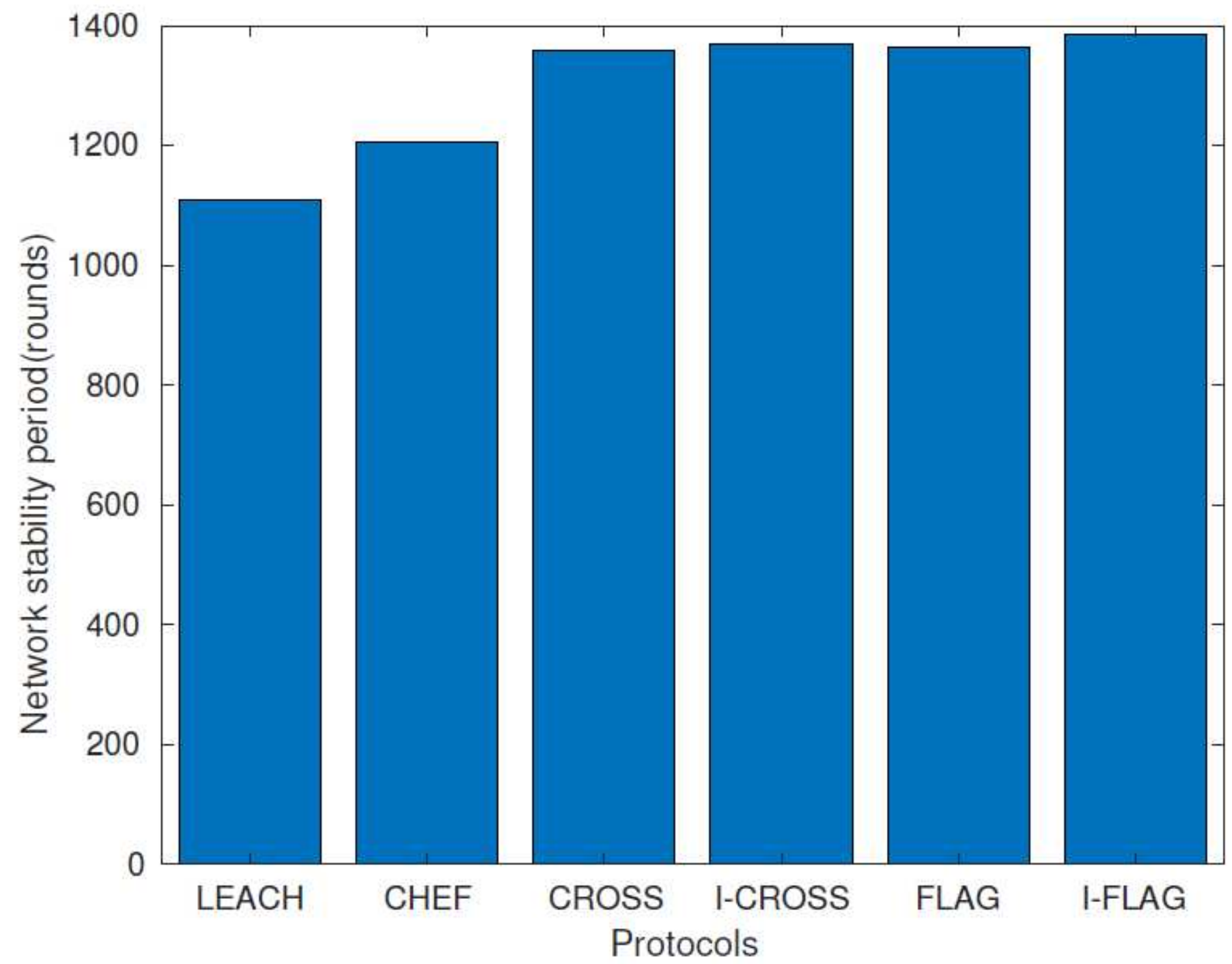

Figure 15

Protocols Vs Network stability period 


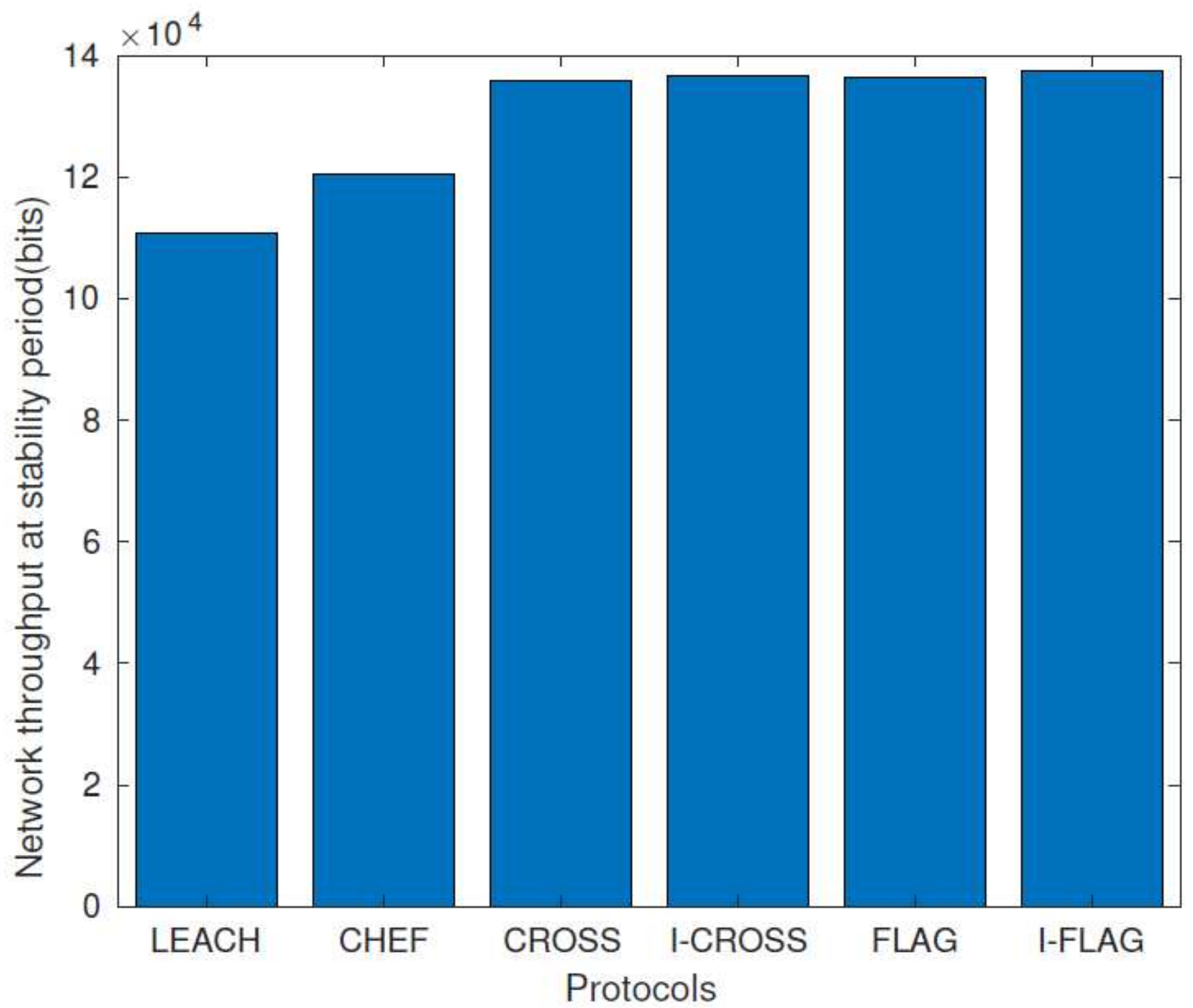

Figure 16

Protocols Vs Network throughput at stability period 